\title{
Nanofluids for Heat Transfer
}

\author{
Rodolphe Heyd \\ CRMD UMR6619 CNRS/Orléans University
}

France

\section{Introduction}

\subsection{A need for energy saving}

The global warming and nuclear or ecological disasters are some current events that show us that it is urgent to better consider renewable energy sources. Unfortunately, as shown by figures of the International Energy Agency (IEA), clean energies like solar, geothermal or wind power represent today only a negligible fraction of the energy balance of the planet. During 2008 , the share of renewable energies accounted for 86 Mtoe, only $0.7 \%$ of the 12,267 Mtoe of global consumption. Unfortunately, the vital transition from fossil fuels to renewable energies is very costly in time and energy, as evidenced by such high costs of design and fabication of photovoltaic panels. Thus it is accepted today that a more systematic use of renewable energy is not sufficient to meet the energy challenge for the future, we must develop other ways such as for example improving the energy efficiency, an area where heat transfers play an important role.

In many industrial and technical applications, ranging from the cooling of the engines and high power transformers to heat exchangers used in solar hot water panels or in refrigeration systems, the low thermal conductivity $k$ of most heat transfer fluids like water, oils or ethylene-glycol is a significant obstacle for an efficient transfer of thermal energy (Table 1).

\begin{tabular}{c|c|c|c|c|c}
\hline liquids: & $\begin{array}{c}\text { Ethylen } \\
\text { Glycol } \\
(\mathrm{EG})\end{array}$ & $\begin{array}{c}\text { Glycerol } \\
(\mathrm{Gl})\end{array}$ & Water $(\mathrm{Wa})$ & $\begin{array}{c}\text { Thermal } \\
\text { Compound } \\
(\mathrm{TC})\end{array}$ & \\
\hline$k(\mathrm{~W} / \mathrm{mK})$ & 0.26 & 0.28 & 0.61 & $\approx 0.9$ & \\
\hline \hline metals: & Iron & Aluminium & Copper & Silver & CNT \\
\hline$k(\mathrm{~W} / \mathrm{mK})$ & 80 & 237 & 400 & 429 & $\approx 2500$ \\
\hline
\end{tabular}

Table 1. Thermal conductivities $k$ of some common materials at RT.

The improvement of heat transfer efficiency is an important step to achieve energy savings and, in so doing, address future global energy needs. According to Fourier's law $\mathbf{j}_{Q}=-k \nabla T$, an increase of the thermal conductivity $k$ will result in an increase of the conductive heat flux. Thus one way to address the challenge of energy saving is to combine the transport properties of some common liquids with the high thermal conductivity of some common metals (Table 1) such as copper or novel forms of carbon such as nanotubes (CNT). These composite materials involve the stable suspension of highly conducting materials in nanoparticulate form to the 
fluid of interest and are named nanofluids, a term introduced by Choi in 1995 (Choi, 1995). A nanoparticle (NP) is commonly defined as an assembly of bounded atoms with at least one of its characteristic dimensions smaller than $100 \mathrm{~nm}$. Due to their very high surface to volume ratio, nanoparticles exhibit some remarkable and sometimes new physical and chemical properties, in some way intermediate between those of isolated atoms and those of bulk material.

\subsection{Some applications and interests of nanocomposites}

Since the first report on the synthesis of nanotubes by Iijima in 1991 (Iijima, 1991), there has been a sharp increase of scientific interest about the properties of the nanomaterials and their possible uses in many technical and scientific areas, ranging from heat exchange, cooling and lubrication to the vectorization of therapeutic molecules against cancer and biochemical sensing or imaging. The metal or metal oxides nanoparticles are certainly the most widely used in these application areas.

It has been experimentally proved that the suspension in a liquid of some kinds of nanoparticles, even in very small proportions ( $<1 \%$ by volume), is capable of increasing the thermal conductivity of the latter by nearly $200 \%$ in the case of carbon nanotubes (Casquillas, 2008; Choi et al., 2001), and approximately $40 \%$ in the case of copper oxide nanoparticles (Eastman et al., 2001). Since 2001, many studies have been conducted on this new class of fluids to provide a better understanding of the mechanisms involved, and thus enable the development of more efficient heat transfer fluids. The high thermal conductivity of the nanofluids designates them as potential candidates for replacement of the heat carrier fluids used in heat exchangers in order to improve their performances. It should be noted that certain limitations may reduce the positive impact of nanofluids. Thus the study of the performance of cooling in the dynamic regime showed that the addition of nanoparticles in a liquid increases its viscosity and thereby induces harmful losses (Yang et al., 2005). On the other hand, the loss of stability in time of some nanofluids may result in the agglomeration of the nanoparticles and lead to a modification in their thermal conduction properties and to risks of deposits as well as to the various disadvantages of heterogeneous fluid-flow, like abrasion and obstruction. Nevertheless, in the current state of the researches, these two effects are less important with the use of the nanofluids than with the use of the conventional suspensions of microparticles (Daungthongsuk \& Wongwises, 2007). We must not forget to take into account the high ecological cost of the synthesis of the NPs, which often involves a large number of chemical contaminants. Green route to the synthesis of the NPs using natural substances should be further developed (Darroudi et al., 2010).

\section{Preparation of thermal nanofluids}

\subsection{Metal nanoparticles synthesis}

\subsubsection{Presentation}

Various physical and chemical techniques are available for producing metal nanoparticles. These different methods make it possible to obtain free nanoparticles, coated by a polymer or encapsulated into a host matrix like mesoporous silica for example. In this last case, they are protected from the outside atmosphere and so from the oxidation. As a result of their very high surface to volume ratio, NPs are extremely reactive and oxidize much faster than in the bulk state. The encapsulation also avoids an eventual agglomeration of the nanoparticles 
as aggregates (clusters) whose physico-chemical properties are similar to that of the bulk material and are therefore much less interesting. The choice of a synthesis method is dictated by the ultimate use of nanoparticles as: nanofluids, sensors, magnetic tapes, therapeutic molecules vectors,etc. Key factors for this choice are generally: the size, shape, yield and final state like powder, colloidal suspension or polymer film.

\subsubsection{Physical route}

The simplest physical method consists to subdivide a bulk material up to the nanometric scale. However, this method has significant limitations because it does not allow precise control of size distributions. To better control the size and morphology, we can use other more sophisticated physical methods such as:

- the sputtering of a target material, for example with the aid of a plasma (cathode sputtering), or with an intense laser beam (laser ablation). K. Sakuma and K. Ishii have synthesized magnetic nanoparticles of Co-Pt and Fe with sizes ranging from 4 to $6 \mathrm{~nm}$ (Sakuma \& Ishii, 2009).

- the heating at very high temperatures (thermal evaporation) of a material in order that the atoms constituting the material evaporate. Then adequate cooling of the vapors allows agglomeration of the vapor atoms into nanoparticles (Singh et al., 2002).

The physical methods often require expensive equipments for a yield of nanoparticles often very limited. The synthesized nanoparticles are mostly deposited or bonded on a substrate.

\subsubsection{Chemical route}

Many syntheses by the chemical route are available today and have the advantage of being generally simple to implement, quantitative and often inexpensive. Metallic NPs are often obtained via the reduction of metallic ions contained in compounds like silver nitrate, copper chloride, chloroauric acid, bismuth chloride, etc.

We only mention here a few chemical methods chosen among the most widely used for the synthesis of metal and metal oxides NPs:

Reduction with polymers: schematically, the synthesis of metal nanoparticles (M) from a solution of $\mathrm{M}^{+}$ions results from the gradual reduction of these ions by a weak reducing polymer (suitable to control the final particle size) such as PVA (polyvinyl alcohol) or PEO (polyethylene oxide). The metal clusters thus obtained are eventually extracted from the host polymer matrix by simple heating. The size of the synthesized metal nanoparticles mainly depends on the molecular weight of the polymer and of the type of metal ions. For example with PVA $\left(M_{w}=10000\right)$ we obtained (Hadaoui et al., 2009) silver nanoparticles with a diameter ranging from 10 to $30 \mathrm{~nm}$ and copper nanoparticles with a diameter of about $80 \mathrm{~nm}$.

Gamma radiolysis: the principle of radiolytic synthesis of nanoparticles consists in reducing the metal ions contained in a solution through intermediate species (usually electrons) produced by radiolysis. The synthesis can be described in three parts (i) radiolysis of the solvent, (ii) reduction reaction of metal ions by species produced by radiolysis followed by (iii) coalescence of the produced atoms (Benoit et al., 2009; Ramnani et al., 2007; Temgire et al., 2011). 
Thermal decomposition: the synthesis by the thermal decomposition of an organometallic precursor allows to elaborate various systems of nanoparticles (Chen et al., 2007; Liu et al., 2007; Roca et al., 2006; Sun et al., 2004) or carbon nanotubes (Govindaraj \& Rao, 2002). This method is widely used because of its ease and of the reproducibility of the synthesis, as well as the uniformity in shape and size of the synthesized particles. Metal particles such as $\mathrm{Au}, \mathrm{Ag}, \mathrm{Cu}, \mathrm{Co}, \mathrm{Fe}, \mathrm{FePt}$, and oxides such as copper oxides, magnetite and other ferrites have been synthesized by this method. It mainly consists of the decomposition of an organometallic precursor dissolved in an organic solvent (like trioctylamine, oleylamine, etc.) with high boiling points and containing some surfactants (so called capping ligands) like oleic acid, lauric acid, etc. By binding to the surface of the NPs, these surfactants give rise to a steric barrier against aggregation, limiting the growing phase of the nanoparticles. Basing on the choice of the ligand properties (molecular length, decomposition temperature) and on the ligand/precursor ratio, it is possible to control the size and size distribution of the synthesized NPs (Yin et al., 2004).

Using the thermal decomposition of the acetylacetonate copper precursor dissolved in oleylamine in the presence of oleic acid, we have synthesized copper oxide nanoparticles of mean diameter $7 \mathrm{~nm}$ with a quasi-spherical shape and low size dispersion (Fig. 1).
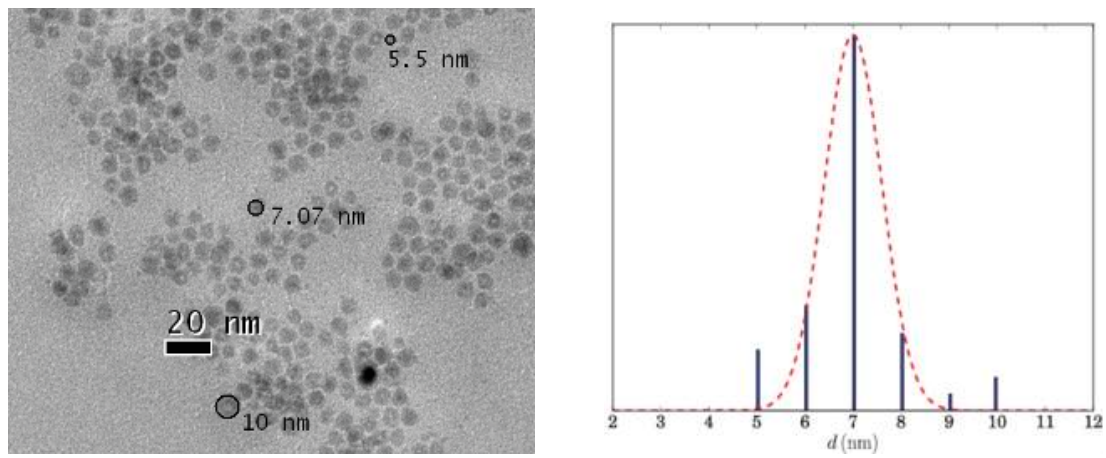

Fig. 1. TEM picture of copper oxides nanoparticles synthesized by the thermal decomposition of acetylacetonate copper precursor dissolved in oleylamine (Hadaoui, 2010).

\subsubsection{Characterization of the nanoparticles}

Depending on the final state of the nanoparticles, there are several techniques to visualize and characterize them: the X-ray diffraction, electron microscopy (TEM, cryo-TEM, etc.), the atomic force microscopy, photoelectron spectroscopy like XPS. More macroscopic methods like IR spectroscopy and UV-visible spectroscopy are interesting too in the case where there is a plasmonic resonance depending on the size of the NPs like for example in the case of silver and gold.

The Dynamic Light Scattering (DLS) is a well established technique to measure hydrodynamic sizes, polydispersities and aggregation effects of nanoparticles dispersed in a colloidal suspension. This method is based on the measurement of the laser light scattering fluctuations due to the Brownian motion of the suspended NPs. In the case of opaque nanofluids, only the backscattering mode of DLS is able to provide informations on NPs characteristics. 


\subsection{Stability of colloidal suspensions}

\subsubsection{Presentation}

The nanofluids belong to the class of Solid/Liquid colloidal systems where a solid phase is very finely dispersed in a continous liquid phase. Most of nanofluids are prepared by direct injection of nanoparticles in the host liquid, depending on the nature of this liquid (water, ethylene glycol (EG), oils, glycerol, etc.) it may be necessary to add chemicals to the solution to avoid coagulation and ensure its stability by balancing internal forces exerted on particles and slowing down agglomeration rates. This addition can dramatically change the physical properties of the base liquid and give disappointing results.

\subsubsection{Isolated spherical particle immersed in a fluid}

We consider a spherical particle of radius $a_{p}$, density $\rho_{p}$, immersed in a fluid of density $\rho_{f}$ and dynamical viscosity $\eta$, placed at rest in the gravitational field $\mathbf{g}$ assumed to be uniform (Fig. 2(a)). Under the effect of its weight $\mathbf{P}=\rho_{p} V_{p} \mathbf{g}$ and of the buoyancy $\mathbf{F}_{A}=-\rho_{f} V_{p} \mathbf{g}$ due to the fluid, the particle moves with velocity $\mathbf{v}$ that obeys to the equation of motion $m_{p} \frac{\mathrm{d} \mathbf{v}}{\mathrm{d} t}=\Delta \mathbf{F}+\mathbf{F}_{v}$, where $\Delta \mathbf{F}=\mathbf{P}+\mathbf{F}_{A}=V_{p}\left(\rho_{p}-\rho_{f}\right) \mathbf{g}$ and $\mathbf{F}_{v}$ is the viscous drag exerted by the fluid on the particle. In the limit of laminar flow at very low Reynolds numbers $\operatorname{Re}=\rho_{f} v 2 a_{p} / \eta \ll 1$, we can write the Stokes law for a sphere as $\mathbf{F}_{v}=-6 \pi a_{p} \eta \mathbf{v}$. We deduce from these hypotheses the following equation satisfied by the velocity of the sphere:

$$
\frac{\mathrm{d} \mathbf{v}}{\mathrm{d} t}+\frac{6 \pi \eta a_{p}}{\rho_{p} V_{p}} \mathbf{v}=\left(1-\frac{\rho_{p}}{\rho_{f}}\right) \mathbf{g}
$$

As we can see from (1), if $\rho_{p}>\rho_{f}$, agglomeration leads to sedimentation and on the other hand if $\rho_{p}<\rho_{f}$ agglomeration leads to skimming. After a characteristic time $\tau=2 \rho_{p} a_{p}^{2} / 9 \eta$ generally very short, the velocity of the sphere reaches a constant limiting value $v_{\ell}$ (Fig. 2(a)) whose magnitude is given by:

$$
v_{\ell}=\frac{2 g\left|\rho_{p}-\rho_{f}\right| a_{p}^{2}}{9 \eta}
$$

Based on previous results, we can preserve the stability of water-based nanofluids by limiting $a_{p}$, that is by limiting the agglomeration of nanoparticles. In the case of viscous host media (like glycerol or gels), stability is generally guaranteed, even for large agglomerates.

\subsubsection{Coagulation of nanoparticles}

\subsubsection{Presentation}

The coagulation between two particles may occur if:

1. the particles are brought close enough from each other in order to coagulate. When a colloid is not stable, the coagulation rate depends of the frequency at which the particles collide. This dynamic process is mainly a function of the thermal motion of the particles, of the fluid velocity (coagulation due to shear), of its viscosity and of the inter-particles forces (colloidal forces).

2. during the collision the energy of the system is lowered by this process. This decrease in energy originates from the forces, called colloidal forces, acting between the particles in suspension. The colloidal forces are mainly composed of electrostatic repulsive forces 


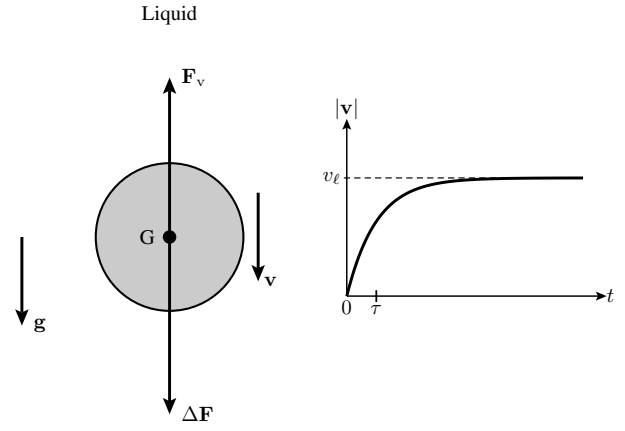

(a) Dynamics of a spherical particle immersed in a liquid at rest.

\begin{tabular}{lll}
\hline radius $a_{p}$ & Water & Glycerol \\
\hline $5 \mathrm{~nm}$ & $400 \mathrm{~d}$ & $420531 \mathrm{~d}$ \\
$50 \mathrm{~nm}$ & $4 \mathrm{~d}$ & $4205 \mathrm{~d}$ \\
$100 \mathrm{~nm}$ & $1 \mathrm{~d}$ & $1051 \mathrm{~d}$ \\
\hline
\end{tabular}

(b) Values (in days) of the time taken by cupric oxide NPs to travel a distance $h=$ $1 \mathrm{~cm}$ in different liquids and for various radii.

Fig. 2. A simple mechanical model to discuss the stability of the nanofluids in the terrestrial gravitational field.

and Van der Waals type forces. The electrostatic forces, always present in the case of water-based nanofluids, are due to the presence of ionised species on the surface of the particles, inducing an electric double layer. More this double-layer is important, the more the particles repel each other and more stable is the solution. The Van der Waals type forces are due to the interactions between the atoms constituing the NPs.

\subsubsection{DLVO theory}

For a system where the electrostatic forces and the Van der Waals forces are dominant, as in the case of water-based nanofluids, the DLVO theory establishes that a simple combination of the two corresponding interaction energies, respectively $U_{e}(s)$ and $U_{\mathrm{VW}}(s)$, is sufficient to explain any tendency to the aggregation of the suspension. This concept was developed by Derjaguin and Landau and also by Vervey and Overbeck.

In the case of two identical interacting spherical particles with radius $a_{p}$, separated by a distance $s$ (Fig. 3), it is possible (Masliyah \& Bhattarjee, 2006) to write the DLVO interaction energy $U(s)$ as:

$$
\begin{aligned}
U(s) & =U_{e}(s)+U_{\mathrm{VW}}(s) \\
& =2 \pi a_{p} \epsilon_{r} \epsilon_{0} \psi_{S}^{2} \ln \left(1+e^{-s / \kappa^{-1}}\right)-\frac{A_{H} a_{p}}{12 s}
\end{aligned}
$$

where $A_{H}$ is the Hamaker's constant $\left(A_{H} \approx 30 \times 10^{-20} \mathrm{~J}\right.$ for copper NPs in water), $\epsilon_{0}$ is the permittivity of vacuum, $\epsilon_{r}$ is the dielectric constant of the host fluid $\left(\epsilon_{r}=78.5\right.$ for water at $\mathrm{RT}), \psi_{S}$ is the surface electrical potential of NPs and $\kappa^{-1}$ is the Debye length defined by:

$$
\kappa^{-1}=\left(\frac{10^{3} N_{A} e^{2}}{\epsilon_{0} \epsilon_{r} k_{B} T} \sum_{i=1}^{N} z_{i}^{2} M_{i}\right)^{-1 / 2}
$$

where $z_{i}$ is the valence of $i^{\text {th }}$ ionic species and $M_{i}$ is its moalrity, $N_{A}$ is the Avogadro number, $k_{B}$ is the Boltzmann constant, $e$ is the elementary charge and $T$ is the absolute temperature 
of the colloid. The Debye length gives an indication of the double layer thickness, thus more $\kappa^{-1}$ is important, better is the stability of the suspension. Introducing the ionic strength $I=$ $\sum_{i=1}^{N} z_{i}^{2} M_{i} / 2$, we see from (4) that using high values of $I$ makes the suspension unstable. It is therefore recommended to use highly deionized water to prepare water-based nanofluids.

As can be seen on Fig. 3(a), the colloidal suspension is all the more stable that there is a significant energy barrier $E_{b}$, preventing the coagulation of nanoparticles.

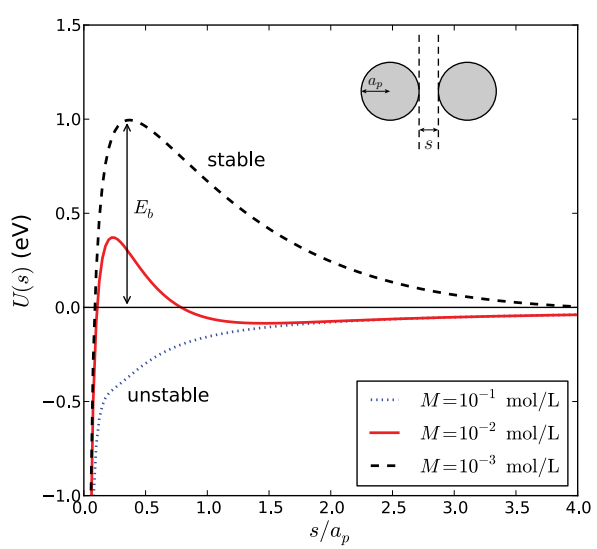

(a) DLVO interaction energy and stability tendencies of copper oxide spherical NPs suspended in water at RT and using symetric electrolytes with different molarities $M$ and $\psi_{S}=0.1 \mathrm{~V}$.

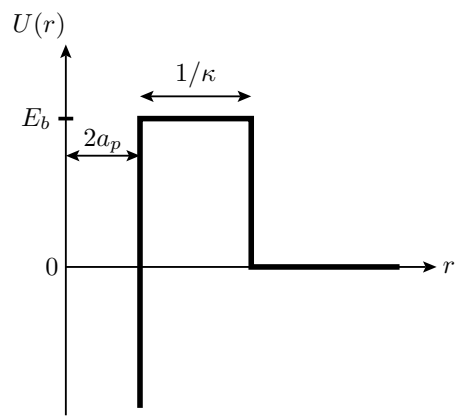

(b) Approximate DLVO interaction potentiel used to calculate the frequency collision function $K^{\prime}$.

Fig. 3. DLVO interaction energy in the case of two identical spheres. We recall that $1 \mathrm{eV} \approx 39 k_{B} T$ at RT and $r=s+2 a_{p}$ is the distance between the centers of two particles.

\subsubsection{Dynamics of agglomeration}

If on the one hand the colloidal forces are a key factor to discuss the stability of a suspension, on the other hand the dynamics of the collisions is another key factor.

We note $J_{k}^{+}>0$ the rate of formation per unit volume of particles of volume $v_{k}$ and $J_{k}^{-}<0$ the rate of disappearance per unit volume. With these notations the net balance equation for the $k^{\text {th }}$ species is written as:

$$
\frac{\mathrm{d} n_{k}}{\mathrm{~d} t}=J_{k}^{+}+J_{k}^{-}
$$

where $n_{k}$ is the number of particles of the $k^{\text {th }}$ species per unit volume. Von Smoluchowski proposed the following expressions of $J_{k}^{+}$and $J_{k}^{-}$to describe the formation of any aggregate of volume $v_{k}$ :

$$
\begin{aligned}
J_{k}^{+} & =\frac{1}{2} \sum_{i=1 ; v_{i}+v_{j}=v_{k}}^{i=k-1} \beta_{i j} n_{i} n_{j} \\
J_{k}^{-} & =-\sum_{i=1}^{N_{p}} \beta_{k i} n_{i} n_{k}
\end{aligned}
$$


where $\beta_{i j}$ is the collision frequency function and $N_{p}$ is the total number of particles species or equivalently of different volumes. The $1 / 2$ factor in (6) takes care of the fact that $v_{i}+v_{j}=$ $v_{j}+v_{i}$. The collision frequency function $\beta_{i j}$ is the key insight of the kinetics of coagulation and is tightly dependent of several factors such as: the Brownian motion (thermal motion) or the deterministic motion (fluid-flow) of the fluid, the nature of the inter-particles forces and of the aggregation ("touch-and-paste" or "touch-and-go", the last case requiring then many collisions before permanent adhesion). If we consider the simplest case of an initially monodisperse colloidal particles modeled as hard spheres and only submitted to Brownian motion, the collision frequency function is given (Masliyah \& Bhattarjee, 2006) by:

$$
K=\beta_{k k}=\frac{8 k_{B} T}{3 \eta}
$$

This simple result shows once again that the use of viscous fluids host significantly slows the onset of aggregation of nanofluids.

We now need an estimate of the time $t_{1 / 2}$ needed for the coagulation for example of one half of the initial population of nanoparticles. For simplicity we suppose that there is only binary collisions of identical particles of kind (1) and volume $v_{1}$. We assume that every collision leads by coagulation to the formation of a particle of kind (2) and volume $v_{2}=2 v_{1}$ and that this particle deposits as a sediment without undergoing another collisions. Using relations (5), (6) and (7) we write:

$$
\begin{aligned}
\frac{\mathrm{d} n_{1}}{\mathrm{~d} t} & =-\beta_{11} n_{1}^{2} \Rightarrow n_{1}(t)=\frac{n_{1}(0)}{1+\beta_{11} n_{1}(0) t} \\
\frac{\mathrm{d} n_{2}}{\mathrm{~d} t}=\frac{1}{2} \beta_{11} n_{1}^{2} \Rightarrow n_{2}(t) & =\frac{1 / 2 \beta_{11} n_{1}^{2}(0) t}{1+\beta_{11} n_{1}(0) t}
\end{aligned}
$$

where $n_{1}(0)$ is the initial number of particles per unit volume. Introducing $t_{1 / 2}=1 / \beta_{11} n_{1}(0)$, recalling that the volume fraction of NPs is written as $\phi=4 / 3 \pi a_{p}^{3} n_{1}(0)$ and using (8), the time $t_{1 / 2}$ can be expressed as:

$$
t_{1 / 2}=\frac{\eta \pi a_{p}^{3}}{2 \phi k_{B} T}
$$

In the case of a water-based nanofluid containing a volume fraction $\phi=0.1 \%$ of identical spherical particles with radius $a_{p}=10 \mathrm{~nm}$, we found with our model that $t_{1 / 2}=0.38 \mathrm{~ms}$ at RT, which is a quite small value! The relation (11) qualitatively shows that it is preferable to use low NPs volume fractions suspended in viscous fluids. For the same volume fractions, small NPs aggregate faster than the bigger.

A more sophisticated approach includes the colloidal forces between particles. Using an approximated DLVO potential of the form represented Fig. 3(b) can lead to the following approximated expression of the frequency collision function $K^{\prime}$ taking into account interactions:

$$
K^{\prime}=2 a_{p} \kappa \exp \left(-\frac{E_{b}}{k_{B} T}\right) K
$$

We will retain from this expression that more the colloidal forces are repulsive $\left(E_{b} / k_{B} T \gg 1\right)$, more the coagulation of particles is slow and the solution is stable over time. 


\subsubsection{How to control aggregation in nanofluids?}

The preceeding studies have shown that, to control the agglomeration of NPs in the suspension and avoid settling, it is recommended to use:

- viscous host fluids with high value of the dielectric constant, low particles volume fraction $\phi$ and not too small particles ;

- pure highly desionized water with low values of the ionic strength $I$ (in the case of water-based nanofluids);

- $\mathrm{pH}$ outside the region of the isoelectric point for the case of amphoteric NPs (like silica and metal oxides) suspended in water. The isoelectric point (IEP) may be defined as the $\mathrm{pH}$ at which the surface of the NP exhibits a neutral net electrical charge or equivalently a zero zeta potential $\zeta=0 \mathrm{~V}$. For this particular value of $\zeta$ there are only attractive forces of Van der Waals and the solution is not stable. For example in the case of copper oxide NPs suspended in water, $\operatorname{IEP}(\mathrm{CuO}) \approx 9.5$ at $\mathrm{RT}$ and a neutral or acid $\mathrm{pH} \leqslant 7$ promotes the stability of the suspension.

- surface coating with surfactants or with low molecular weight $\left(M_{w}<10000\right)$ neutral polymers highly soluble in the liquid suspension. They allow to saturate the surface of NPs without affecting the long range repulsive electrostatic force. In contrast this polymeric shell induces steric effects that may dominate the short distances attractive Van der Waals interaction. Thus forces are always repulsive and the solution is stable. In a sense the presence of the polymer shell enhances the value of the energy barrier $E_{b}$.

- high power sonication to break agglomerates and disperse particles.

It is important to mention here that the surface treatments we presented above allow to enhance the stability of the suspension and to control the aggregation, but unfortunately they certainly also have a deep impact on the heat transfer properties of the nanofluid and should be considered carefully. The control of the NPs surface using polymer coating, surfactants or ions grafting, introduces unknown thermal interfacial resistances which can dramatically alter the benefit of using highly conductive nanoparticles.

\section{Thermal transfer coefficients of nanofluids}

\subsection{Presentation}

The use of suspended nanoparticles in various base fluids (thermal carriers and biomedical liquids for example) can alter heat transfer and fluid flow characteristics of these base fluids. Before any wide industrial application can be found for nanofluids, thorough and systematic studies need to be carried out. Apart of the potential industrial applications, the study of the nanofluids is of great interest to the understanding of the mechanisms involved in the processes of heat transfer to the molecular level. Experimental measurements show that the thermal properties of the nanofluids do not follow the predictions given by the classical theories used to describe the homogeneous suspensions of solid micro-particles in a liquid. Despite the large number of published studies on the subject in recent years, today there is no unique theory that is able to properly describe the whole experimental results obtained on the nanofluids. 


\begin{tabular}{|c|c|c|c|}
\hline Fluids & Particles, size $(\mathrm{nm})$ & $\phi(\%)$ & Improvement (\%) \\
\hline$\overline{\mathrm{EG}}$ & $\mathrm{CuO}, 18.6$ & 4 & 20 \\
\hline water & $\mathrm{CuO}, 18.6$ & 4.3 & 10 \\
\hline GL & $\mathrm{Cu}_{2} \mathrm{O}, 7.0$ & 0.6 & 120 \\
\hline GL & $\mathrm{Cu}_{2} \mathrm{O}, 150$ & 0.6 & 60 \\
\hline EG & $\mathrm{Cu}, 10$ & 0.2 & 40 \\
\hline $\mathrm{PO}$ & $\mathrm{Cu}, 35$ & 0.06 & 45 \\
\hline water & $\mathrm{Cu}, 100$ & 7.5 & 75 \\
\hline water & $\mathrm{TiO}_{2}, 15$ & 4 & 33 \\
\hline water & $\mathrm{TiO}_{2}, 27$ & 4.3 & 10.6 \\
\hline water & $\mathrm{Al}_{2} \mathrm{O}_{3}, 60$ & 5 & 20 \\
\hline EG & $\mathrm{Al}_{2} \mathrm{O}_{3}, 60$ & 5 & 30 \\
\hline $\mathrm{PO}$ & $\mathrm{Al}_{2} \mathrm{O}_{3}, 60$ & 5 & 40 \\
\hline water & $\mathrm{Al}_{2} \mathrm{O}_{3}, 10$ & 0.5 & 100 \\
\hline water & $\mathrm{Al}_{2} \mathrm{O}_{3}, 20$ & 1 & 16 \\
\hline oil & MWCNTs, 25 & 1.0 & $\geqslant 250$ \\
\hline water & MWCNTs, 130 & 0.6 & 34 \\
\hline
\end{tabular}

Table 2. Some significant results relating to the improvement of the thermal conductivity of nanofluids at RT. PO: pump oil; EG: ethylene glycol; GL: glycerol

\subsection{Experimental results}

Since the pioneering works of Choi, many experimental studies have been conducted on thermal nanofluids and have shown very large dispersion in the results. There is a profusion of very varied experimental results, sometimes contradictory, so it is very difficult for the novice and sometimes even for the specialist to identify a trend in the contribution of thermal nanofluids for heat transfer. We have gathered in Tab. 2 some of the the most significant results published to date on the improvement of the thermal conductivity of nanofluids containing metallic particles, oxides particles or CNTs. We can identify several trends and indications

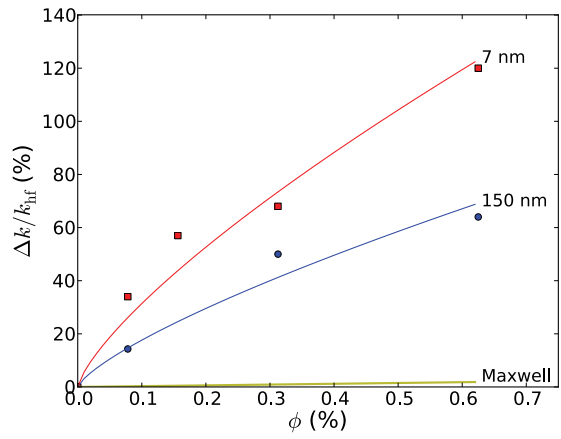

(a) Enhancement as a function of NPs size and volume fraction in the case of $\mathrm{Cu}_{2} \mathrm{O} /$ glycerol nanofluid.

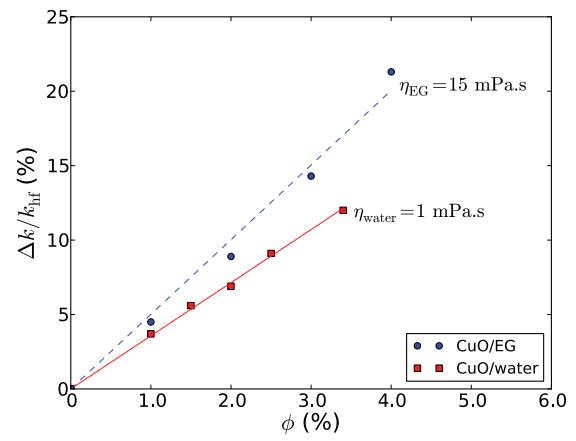

(b) Enhancement as a function of host fluid viscosity and volume fraction.

Fig. 4. Thermal conductivity enhancement of nanofluids at RT. $k_{\mathrm{hf}}$ is the thermal conductivity of the host fluid at RT. 
from the preceding experimental results:

- For the same volume concentrations, the improvement of thermal conductivity $\Delta k / k_{\mathrm{hf}}$ obtained with NPs suspensions is much higher than that obtained with equivalent suspensions of micro-particles. The classical laws such as Maxwell-Garnett or Hamilton-Crosser (Tab. 3) are no longer valid in the case of nanofluids (Fig. 4(a)).

- The size $d$ of the nanoparticles has a moderate influence on the improvement of the thermal conductivity. The more the NPs are smaller, the more the increase is significant (Fig. 4(a), Tab. 2). This behavior is not predictable using the classical laws of table 3.

- The viscosity of the host fluid also appears to play a significant role that has not been sufficiently explored so far. As shown by the measurements taken at room temperature with $\mathrm{Al}_{2} \mathrm{O}_{3}$ in various liquids (water, EG and oil) and the measurements of figure 4(b) about $\mathrm{CuO}$, the improvement of the thermal conductivity increases with the viscosity of the host fluid.

- The nature of the particles and host fluid also plays an important role. However it is very difficult to identify clear trends due to the various NPs surface treatments (surfactants, polymer coating, $\mathrm{pH}$ ) used to stabilize the suspensions according to the different kinds of interactions NP/fluid and their chemical affinity. Thus we can assume that the surfactants and polymer coatings can significantly modify the heat transfer between the nanoparticles and the fluid.

\subsection{Theoretical approaches}

\subsubsection{Classical macroscopic approach}

As mentioned previously, the conventional models (Tab. 3) do not allow to describe the significant increase of the thermal conductivity observed with nanofluids, even at low volume fractions. These models are essentially based on solving the stationary heat equation $\nabla(k \nabla T)=0$ in a macroscopic way. By using metallic particles or oxides, one may assume that $\alpha=k_{\mathrm{p}} / k_{\mathrm{hf}} \gg 1$ (large thermal contrast). Under these conditions, one can write from the (MG) mixing rule: $\phi_{\mathrm{MG}} \approx 1 /\left(1+3 k_{\mathrm{hf}} / \Delta k\right)$. In the case of copper nanoparticles suspended in pump oil at RT (Table 2), it was found that $\Delta k / k_{\mathrm{hf}}=0.45$ for $\phi_{\exp }=0.06 \%$ while the corresponding value provided by $(\mathrm{MG})$ is $\phi_{\mathrm{MG}} \approx 13 \%$, ie 200 times bigger. These results clearly show that the macroscopic approach is generally not suitable to explain the improvement of thermal conductivity of the thermal nanofluids.

\subsubsection{Heat transfer mechanisms at nanoscale/new models}

We now present the most interesting potential mechanisms allowing to explain the thermal behavior of nanofluids, which are: Brownian motion, ordered liquid layer at the interface between the fluid and the NP, agglomeration across the host fluid.

\subsubsection{Influence of Brownian motion}

The Brownian motion (BM) of the NPs, due to the collisions with host fluid molecules, is frequently mentioned as a possible mechanism for improving the thermal conductivity of nanofluids. There are at least two levels of interpretation:

1. BM induces collisions between particles, in favor of a thermal transfer of solid/solid type, better than that of the liquid/solid type (Keblinski et al., 2002). To discuss the validity of this assumption, we consider the time $\tau_{D}$ needed by a NP to travel a distance $L$ into 


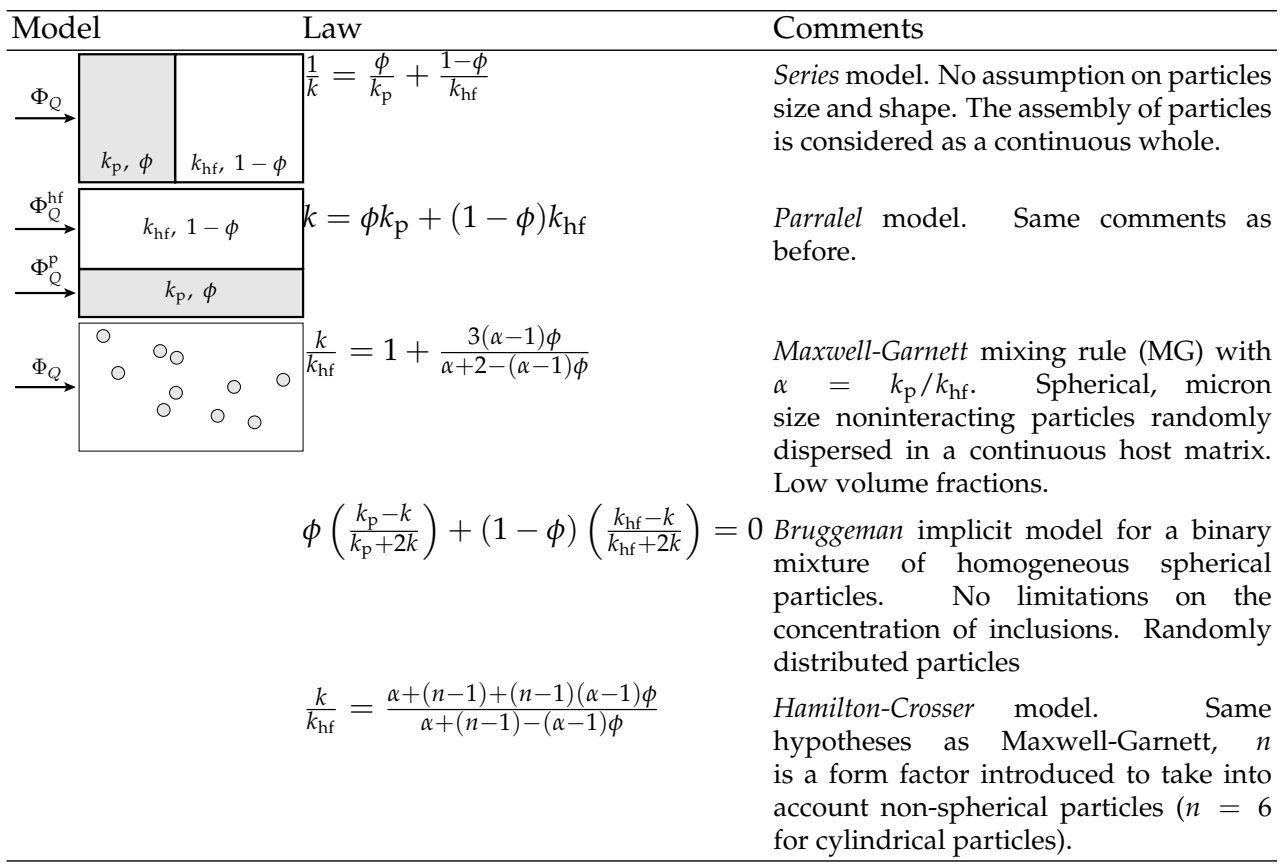

Table 3. Classical models used to describe the thermal conductivity $k$ of micro-suspensions. $k_{\mathrm{p}}$ and $k_{\mathrm{hf}}$ are respectively the thermal conductivities of the particles and of the host fluid, $\phi$ is the volume fraction of particles and $\Phi_{Q}$ is the heat flux.

the fluid due to the Brownian motion. According to the equation of diffusion $\partial n / \partial t-$ $\nabla(D \nabla n)=0$, this time is of order of $\tau_{D}=L^{2} / D$, where $D=k_{B} T / 6 \pi \eta a_{p}$ is the diffusion coefficient for a spherical particle of radius $a_{p}$. Considering now the heat transfer time $\tau_{L}$ associated to heat diffusion in the liquid, we obtain from the heat transfer equation $\rho_{f} \mathcal{c}_{m} \partial T / \partial t-\nabla\left(k_{\mathrm{hf}} \nabla T\right)=0$ in a liquid at rest: $\tau_{L}=L^{2} / \alpha=\rho_{f} \mathcal{c}_{m} L^{2} / k_{\mathrm{hf}}$. The ratio of $\tau_{D} / \tau_{L}$ is given by:

$$
\frac{\tau_{D}}{\tau_{L}}=\frac{3 \pi k_{\mathrm{hf}} \eta a_{p}}{\rho_{f} c_{m} k_{B} T}
$$

For water at room temperature $\left(\eta=10^{-3}\right.$ Pa.s, $\rho_{f}=10^{3} \mathrm{~kg} / \mathrm{m}^{3}, k_{\mathrm{hf}}=0.58 \mathrm{~W} / \mathrm{m} . \mathrm{K}$, $c_{m}=4.18 \mathrm{~kJ} / \mathrm{kg}, k_{B}=1.3810^{-23} \mathrm{~J} / \mathrm{K}$ ) and with $a_{p}=5 \mathrm{~nm}$, Eq. (13) gives $\tau_{D} / \tau_{L} \approx 3000$. This result shows that the transport of heat by thermal diffusion in the liquid is much faster than Brownian diffusion, even within the limit of very small particles. Thus the collisions induced by BM cannot be considered as the main responsible for the significant increase in thermal conductivity of the nanofluids.

2. BM induces a flow of fluid around the nanoparticles, in favor of an additional heat transfer by Brownian forced micro-convection (Wang et al., 2002). To compare the efficiency of the forced convective heat transfer to the heat transfer by conduction, we express the Nusselt number $\mathrm{Nu}$ for a sphere as (White, 1991):

$$
\mathrm{Nu}=2+0.3 \operatorname{Re}^{0.6} \operatorname{Pr}^{1 / 3}=2+\Delta \mathrm{Nu}
$$


where $\operatorname{Re}=2 \rho_{f} v_{\mathrm{BM}} a_{p} / \eta$ is the Reynolds number of the flow around a spherical nanoparticle of radius $a_{p}$ and $\operatorname{Pr}=\eta c_{m} / k_{\mathrm{hf}}$ is the Prandtl number of the host fluid. In the limiting case where there is no flow, $\Delta \mathrm{Nu}=0$. Following Chon (Chon et al., 2005), the average Brownian speed of flow is expressed as $v_{\mathrm{BM}}=D / \bar{\ell}_{\mathrm{hf}}$ where $\bar{\ell}_{\mathrm{hf}}$ is the mean free path of the host fluid molecules and again $D=k_{B} T / 6 \pi \eta a_{p}$. If we suppose that the mean free path of water molecules in the liquid phase is of the order of $\bar{\ell}_{\mathrm{hf}} \approx 0.1 \mathrm{~nm}$ at RT, we find $\Delta \mathrm{Nu} \approx 0.09$, which is negligible. Once again, the forced micro-convection induced by $\mathrm{BM}$ cannot be considered as the main responsible mechanism.

The preceding results show that the Brownian motion of nanoparticles can not be considered as the main responsible for the significant increase in thermal conductivity of the nanofluids.

\subsubsection{Ordered liquid layer at the NP surface}

In solids heat is mainly carried by phonons, which can be seen as sound waves quanta. The acoustic impedances of solids and liquids are generally very different, which means that the phonons mostly reflect at the solid/liquid interface and do not leave the NP. If some phonons initiated in a NP could be emitted in the liquid and remain long enough to reach another particle, this phonon mediated heat transport could allow to explain the increase of thermal conductivity observed for nanofluids. But unfortunately liquids are disordered and the phonon mean free path is much shorter in the liquid that in the solid. The only solution for a phonon to persist out of the NP is to consider an ordered interfacial layer in the liquid in which the atomic structure is significantly more ordered than in the bulk liquid (Henderson \& van Swol, 1984; Yu et al., 2000).

We write the effective radius $a_{p}^{\text {eff }}=a_{p}+e_{\mathrm{L}}$ of the NP ( $e_{\mathrm{L}}$ is the width of the layer) as $a_{p}^{\text {eff }}=\beta^{1 / 3} a_{p}$. The effective volume fraction of the NPs is then given by $\phi^{\text {eff }}=\beta \phi$. Using the approximated MG expression introduced in Par. 3.3.1, we can write the new volume fraction $\phi_{\mathrm{MG}}^{\prime}$ needed to obtain an enhancement $\Delta k / k_{\mathrm{hf}}$ taking into account the ordered liquid layer as:

$$
\phi_{\mathrm{MG}}^{\prime}=\frac{1}{\beta} \frac{1}{1+3 k_{\mathrm{hf}} / \Delta k}=\frac{\phi_{\mathrm{MG}}}{\beta}
$$

If we suppose that $a_{p}^{\text {eff }}=2 a_{p}$, which is a very optimistic value, we obtain $\beta=8$. Thus, taking into account the liquid layer at the solid/liquid interface could permit in the best case to obtain an improvement of one order of magnitude, which is not sufficient to explain the whole increase of the thermal conductivity.

\subsubsection{Influence of clusters}

It has been reported in a benchmark study on the thermal conductivity of nanofluids (Buongiorno et al., 2009) that, the thermal conductivity enhancement afforded by the nanofluids increases with increasing particle loading, with particle aspect ratio and with decreasing basefluid thermal conductivity. This observations seem to be an indirect proof of the role of the aggregation and thus of ordered layer assisted thermal percolation in the mechanisms that could explain the thermal conductivity of nanofluids. As we have seen with glycerol based nanofluids, a large thermal conductivity enhancement (Fig. 4(a)) is accompanied by a sharp viscosity increases (Fig. 11(b)) even at low $(\phi<1 \%)$ nanoparticle volume fractions, which may be indicative of aggregation effects. In addition, some authors (Putnam et al., 2006; Zhang et al., 2006) have demonstrated that nanofluids exhibiting good dispersion generally do not show any unusual enhancement of thermal conductivity. 
By creating paths of low thermal resistance, clustering of particles into local percolating patterns may have a major effect on the effective thermal conductivity (Emami-Meibodi et al., 2010; Evans et al., 2008; Keblinski et al., 2002). Moreover if one takes into account the possibility of an ordered liquid layer in the immediate vicinity of the particle, it can allow a rapid and efficient transfer of thermal energy from one particle to another without any direct contact, avoiding thus large clusters and the settling. Thus the association of local clustering and ordered liquid layer can be the key factor to explain the dramatic enhancement of the thermal conductivity of the nanofluids.

\subsection{Measurement methods}

Over the years many techniques have been developed to measure the thermal conductivity of liquids. A number of these techniques are also used for the nanofluids. In Fig. 5 we have gathered a basic classification, adapted from Paul (Paul et al., 2010), of the main measurement techniques available today. There are mainly the transient methods and the steady-state methods. Compared to solids, measurement of the thermal properties of nanofluids poses

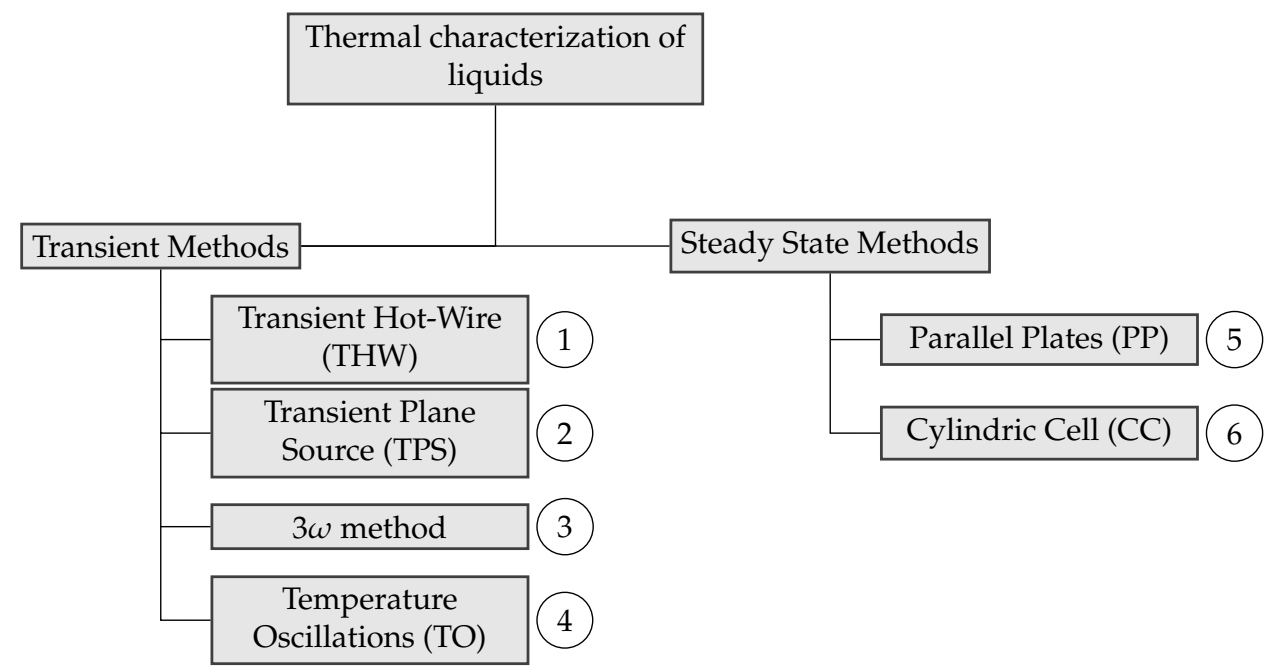

Fig. 5. Different thermal characterization techniques used for nanofluids. The numbers indicate the frequency of occurrence in publications.

many additional issues such as the occurrence of convection, occurrence of aggregates and sedimentation, etc. In the case of the THW method and $3 \omega$ method, which are commonly used and relatively easy to implement, conductive end effects are supplementary problems to take into account. To avoid the influence of convection, sedimentation and conductive end-effects on the measurements it is important that the time $t_{\mathrm{m}}$ taken to measure $k$ is both small compared to the time $t_{\mathrm{cv}}$ of occurence of convection, compared to the time $t_{\mathrm{se}}$ of occurence of sedimentation and compared to the time $t_{\mathrm{ce}}$ of occurence of conductive end-effects influence. There are several solutions to ensure that $t_{\mathrm{m}} \ll t_{\mathrm{cv}}, t_{\mathrm{se}}, t_{\mathrm{ce}}$ :

Convection will occur if the buoyant force resulting from the density gradient exceeds the viscous drag of the fluid, consequently the low viscosity fluids such as water are more 
prone to free convection than more viscous fluids such as oils or ethylen-glycol. To ensure that $t_{\mathrm{m}} \ll t_{\mathrm{cv}}$ it is preferable to:

- limit the rise $\delta T=T(M, t)-T_{i}$ in fluid temperature $T(M, t)$ due to thermal excitation at a low value $\delta T \ll T_{i}$ on the whole domain, with $T_{i}$ the measurement temperature of the fluid. It should be noted that small increases in fluid temperature also limit the energy transfer by radiation.

- use the low viscosity fluids with either a thickener (such as sodium alginate or agar-agar for water) or a flow inhibitor such as glass fiber. These additions should be set to a minimum so as not to significantly change the thermal properties of the examined nanofluids. If the addition of thickeners, even at minimum values, considerably alters the thermal properties of a nanofluid, it could be very interesting to measure these properties in zero-gravity conditions.

- use the most suitable geometry to limit the influence of convection. In the case of plane geometry, it is preferable to heat the liquid by above rather than by below. In the case of heating by hot wire, vertical positioning is a better choice than horizontal.

Conductive end-effects due to electrical contacts are unavoidable but can be limited, when possible, by using a very long heating wire.

Sedimentation will occur if the suspension is not stable over the time. Settling causes a decrease in particle concentration and thermal conductivity. Under these conditions the measurement of the thermal conductivity of nanofluids is not feasible. It is recommended in this case to implement the remarks of paragraph 2.2.3.4.

\subsection{THW and $3 \omega$ methods}

\subsubsection{Presentation}

THW and $3 \omega$ methods are transient techniques that use the generation of heat in the fluid by means of the Joule heating produced in a thin metallic line put in thermal contact with the sample. One then measures the temporal variation $\delta T_{\mathrm{w}}(t)$ of the temperature of the metallic line that results from the thermal excitation, via the variation of its electrical resistance $\delta R(t)$. The more the thermal conductivity of the surrounding liquid is high, the less the increase in temperature of the immersed heating wire is important. This principle is used to measure the thermal conductivity of the liquid to be characterized. Transient techniques have the following advantages:

- They are generally much faster (few minutes) than the quasi-static methods, thus allowing limiting the influence of convection on the measurements.

- They can allow to determine both the thermal conductivity $k$ and specific heat $c_{m}$ of the medium to be characterized.

- The heater is used both as the source of thermal excitation and as the thermometer, thereby eliminating the difficult problem of precise relative positioning of the sensor and the heat source.

- The informative signals are electric which greatly facilitates the design of the instrumentation, of its interface and allows easy extraction and automatic treatment of data.

- The ranges of thermal conductivity measurements can be significant: $0.01 \mathrm{~W} / \mathrm{mK}$ to 100 $\mathrm{W} / \mathrm{mK}$. 
Obviously these methods also have some inconveniences, however few in number:

- The ratio of the length $L$ of the wire to its diameter $d$ should preferably satisfy the relation $L / d \gg 1$ in order to minimize the errors due to the boundary effects of the electrical contacts and convection. This constraint is not easy to achieve when one must characterize very small samples.

- The sample to be characterized has to be an electrical insulator to ensure that electrical current $i(t)$ does not penetrate the fluid. In the case of electrical conducting liquids, it is possible to use metallic wires coated with a thin sheath made of teflon or kapton.

The theoretical basis of these two methods relies on the same theory of an infinite line heat source developed by Carslaw and Jaeger (Carslaw \& Jaeger, 1959). We now present the assumptions of the ideal model of line heater. An infinitely long and infinitely thin line heat source, conductive of electric current, is immersed in an infinite medium at rest whose thermal conductivity has to be measured. The wire and the medium are assumed to be in perfect thermal contact and their physical properties are assumed to be constant. We suppose that the heat is applied in a continuous way between times $t=0$ and $t$. With these assumptions, the temperature rise of the medium satisfies the following expression:

$$
\delta T(r, t)=\frac{1}{4 \pi k} \int_{0}^{t} \dot{q}\left(t^{\prime}\right) e^{-\frac{r^{2}}{4 \alpha\left(t-t^{\prime}\right)}} \frac{\mathrm{d} t^{\prime}}{t-t^{\prime}}
$$

where $\dot{q}$ is the heat rate per unit length $(\mathrm{W} / \mathrm{m}), \alpha=k / \rho c_{m}$ is the thermal diffusivity $\left(\mathrm{m}^{2} / \mathrm{s}\right)$ of the medium and $r$ is the distance from the line at which temperature is measured. A platinum wire is frequently used as the heat line because of its very low reactivity and high electrical conductivity.

\subsubsection{Transient hot wire technique}

\subsubsection{Ideal model}

At initial time $t=0$, the wire is submitted to an abrupt electrical pulse that heats the medium by Joule effect. If we note $\hat{I}$ the constant amplitude of the current intensity flowing across the wire, the rate of heat per unit length can be written as $\dot{q}=R \hat{I}^{2} / L$ where $L$ is the length of the wire in contact with the medium. Of course, the electrical resistance of a metallic wire is a function of temperature and can be writen as $R=R_{\text {ref }}\left[1+\alpha_{w}\left(T-T_{\text {ref }}\right)\right]=R\left(T_{i}\right)+$ $\alpha_{w} R_{\text {ref }} \delta T$, where $\alpha_{w}$ is the temperature coefficient of the wire which is constant in a small range of variation around $T_{\text {ref }}$ and $T_{i}$ is the measurement temperature of the fluid far from the wire. If the amplitude of the electrical pulse is small enough to ensure that $\delta T \ll T_{i}$ then we can make the linear approximation that consists to write the heat rate per unit length as $\dot{q}=R\left(T_{i}\right) \hat{I}^{2} / L=$ cst. According to (16) and by virtue of the temperature continuity across the surface $r=a$ between the wire and the medium, the temperature rise of the infinitely long heat line can be written as:

$$
\delta T(t)=\frac{\dot{q}}{4 \pi k} \int_{0}^{t} e^{-\frac{a^{2}}{4 \alpha\left(t-t^{\prime}\right)}} \frac{\mathrm{d} t^{\prime}}{t-t^{\prime}}=-\frac{\dot{q}}{4 \pi k} \operatorname{Ei}\left(-\frac{a^{2}}{4 \alpha t}\right)
$$

where Ei is the exponential integral, defined (Abramowitz \& Stegun, 1970) for negative argument by:

$$
-\operatorname{Ei}(-x)=\int_{x>0}^{\infty} \frac{e^{-t}}{t} \mathrm{~d} t
$$


where $x=a^{2} / 4 \alpha t$. In real situations, if the condition $L \gg a$ is satisfied, then the expression (17) gives the temperature of the whole wire with a very good approximation as long as convection and boundaries heat conductive losses can be neglected. For times verifying $t \gg t_{\ell}=a^{2} / 4 \alpha$, Eq. (17) can be approximated as:

$$
\delta T(t) \approx \frac{\dot{q}}{4 \pi k}\left(\ln \frac{t}{t_{\ell}}-\gamma\right)
$$

in which $\gamma=0.57722 \ldots$ is Euler's constant. As we can see on Fig. 6(a), a semi-log graph of $\delta T$ versus $\ln t$ or $\log t$ becomes a straight line (dashed line) for $t \gg t_{\ell}$, with a slope proportional to $\dot{q} / 4 \pi k$. The thermal conductivity can be computed from points 1 and 2 belonging to this straight line as:

$$
k=\frac{\dot{q}}{4 \pi} \frac{\ln t_{2} / t_{1}}{\delta T_{2}-\delta T_{1}}
$$

On the other hand, for any time $t \gg t_{\ell}$, points that deviate from the straight line in semi-log scale attest to the onset of the intrinsic limitations of the method such as convection and thermal conduction boundary effects due to the electrical contacts. View the brevity (few

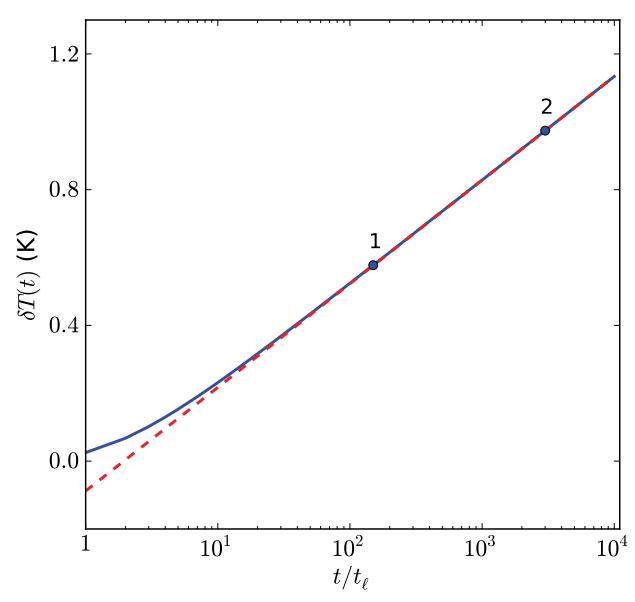

(a) Lin-log plot of $\delta T$ versus $t$ in the case of the ideal model.

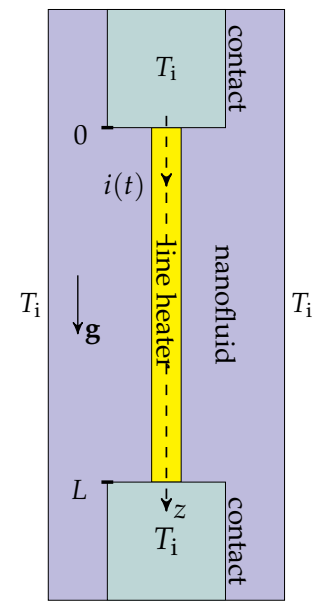

(b) Physical model used for the characterization of nanofluids.

Fig. 6. Exact variation (continuous line) of the temperature rise $\delta T(t)$ of the wire in the case of pure water with $\dot{q}=1 \mathrm{~W} / \mathrm{m}, a=25 \mu \mathrm{m}$ and using the THW technique. The amplitude of $\delta T$ satisfies to the linear approximation.

seconds) of the measurements within the transient technique framework, it is important to quantify the effect of the condition $t \gg t_{\ell}$ on the accuracy of the method. We have gathered in Table 4 the values of $t_{\ell}$ for some common materials and a heater with a radius $a=25 \mu \mathrm{m}$. In the case of water and glycerol, two liquids commonly used as a host fluids for nanoparticles, the relative error using the approximate expression (19) is less than $1 \%$ for measurement times greater than $100 t_{\ell} \approx 0.2 \mathrm{~s}$. 


\begin{tabular}{c|c|c|c|c|c}
\hline Materials & Air & Water & Glycerol & Silicon & Platinum \\
\hline$\alpha \times 10^{5}\left(\mathrm{~m}^{2} . \mathrm{s}^{-1}\right)$ & 1.99 & $1.41 \times 10^{-2}$ & $9.24 \times 10^{-3}$ & 8.94 & 2.51 \\
\hline$t_{\ell}(\mathrm{s})$ & $7.8 \times 10^{-6}$ & $1.1 \times 10^{-3}$ & $1.7 \times 10^{-3}$ & $1.7 \times 10^{-6}$ & $6.2 \times 10^{-6}$ \\
\hline
\end{tabular}

Table 4. Values of $t_{\ell}$ for some common materials at RT.

\subsubsection{Measuring circuit}

The measurement of the temperature rise $\delta T$ of the wire is achieved through the accurate measurement of its small resistance variation $\delta R$. There are mainly two kinds of circuits, the one that uses the classic Wheatstone bridge (Fig. 7) and another that uses a voltage divider.

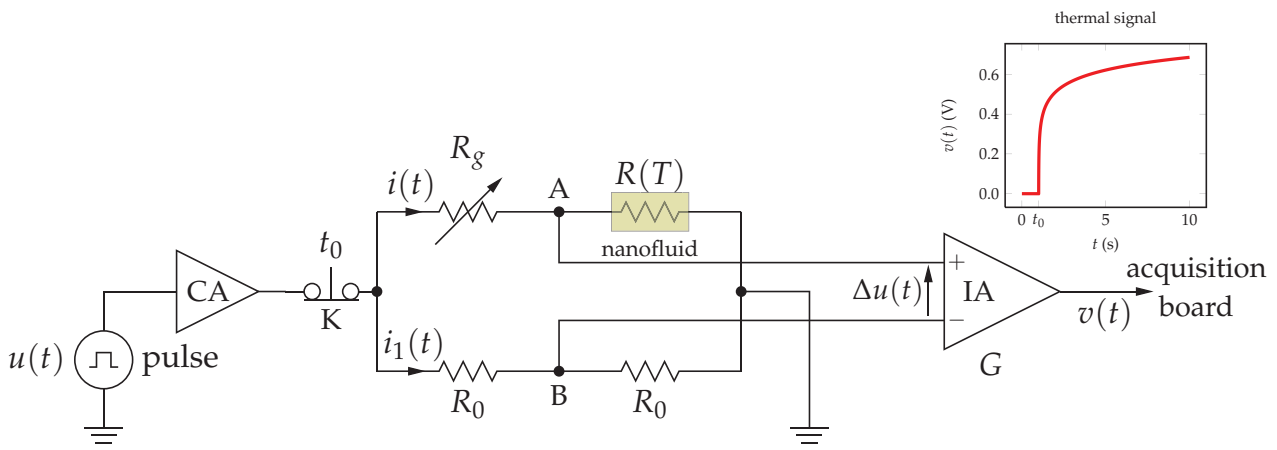

Fig. 7. THW circuit using a Wheatstone bridge.

The $R_{0}$ resistors are chosen such that $i_{1} \ll i . R_{g}$ and $R_{0}$ have a very low temperature coefficient compared to the one of $R(T)$. CA is a low distorsion current buffer (like LT1010) and IA is an instrumentation amplifier (like AD620 or INA126). For each measurement temperature $T_{i}$, the bridge must first be balanced by ensuring that $R_{g}=R\left(T_{i}\right)$. The voltage $v(t)$ delivered by IA is then a function of the temperature rise:

$$
v(t)=\alpha_{w} \frac{G}{4} \frac{R_{\mathrm{ref}}}{R\left(T_{i}\right)} u(t) \delta T(t)
$$

At the initial time $t_{0}$, the switch $\mathrm{K}$ is closed, the current buffer CA imposes through the heating line an electric current of constant intensity $i(t)=\hat{I}$ and thus a constant heat rate per unit length $\dot{q}=R\left(T_{i}\right) \hat{I}^{2} / L$.

\subsubsection{Influence of convection and electrical contacts}

In practice, the main deviations from the law (19) are caused by natural convection and heat conduction at electrical contacts.

Influence of convection: the difference between the temperature of the wire and that of the fluid far from the wire generates a density gradient in the fluid. This density gradient is then the "engine" of a phenomenon of natural convection that takes place within the system. The convection redistributes the thermal energy in the vicinity of the wire in a quite complex manner. The overall impact of this redistribution is a cooling of the wire that results in an overestimated measure of the thermal conductivity of the fluid. 
Influence of electrical contacts: the electrical contacts between the wire and the pulse generator act as heat sinks that cause further cooling of the wire, which also results in an overestimation of the thermal conductivity of the fluid.

As we can see, the convection and electrical contacts lead to an overestimation of the thermal conductivity that can be significant. These two phenomena are not independent and we have to calculate their influence as realistically as possible. To evaluate the effects of the convection and electrical contacts on the measures within the framework of the transient techniques, we have numerically solved the heat and Navier-Stokes equations of the system, mutually coupled by a term of natural convection.

We note $\mathbf{u}$ the eulerian velocity field of the fluid, $\eta$ is the dynamic viscosity and the pressure $P$ is written as $P=p+\rho_{0} g z$. Using the Boussinesq approximation and considering that the fluid is newtonian, the Navier-Stokes equation (NS) is written as:

$$
\text { (NS) }: \rho_{0} \frac{\partial \mathbf{u}}{\partial t}+\rho_{0} \mathbf{u} \cdot \nabla \mathbf{u}=-\nabla p+\eta \nabla^{2} \mathbf{u}-\beta \rho_{0} \mathbf{g} \delta T
$$

where $\beta$ is the coefficient of thermal expansion of the fluid, related to its density variation $\delta \rho$ by the relation $\delta \rho=-\beta \rho_{0} \delta$ T and $\rho_{0}=\rho\left(T_{i}\right)$ is the density of the fluid in absence of themal excitation. The heat equation (HE) for a flowing fluid without a source term is written as:

$$
(\mathrm{HE}): \rho_{0} c_{m} \frac{\partial T}{\partial t}-k \nabla^{2} T=-\rho_{0} c_{m} \mathbf{u} \cdot \nabla T
$$

Finally it remains to express the material balance $(\mathrm{MB})$ for an incompressible fluid:

$$
(\mathrm{MB}): \nabla \cdot \mathbf{u}=0
$$

Denoting $\partial \Omega$ the frontier delimiting the fluid, the set of boundary conditions that accompanies the system of differential equations (NS, HE, MB) satisfied by the fluid is as follows:

$$
T=T_{i} \text { and } \mathbf{u}=\mathbf{0}
$$

exept at the wire interface where the temperature and heat flux are continuous.

There is no exact solution of this system of coupled equations with the set of boundary conditions (25). To our knowledge, the numerical resolution of this system has not yet been explored in order to clarify the influence of the convection and thermal contacts on the accuracy of the measurements in the case of the transient methods. Knibbe is the only one to have explored a similar set of equations for the same purpose but assuming an infinite wire and a decoupling between the thermal conduction and convection (Knibbe, 1986). We note $\delta T_{\mathrm{id}}, \delta \mathrm{T}_{\mathrm{ec}}$ and $\delta \mathrm{T}_{\text {tot }}$ the variations of the wire temperature respectively in the ideal case of an infinite wire without convection given by (19), due to the electrical contacts only and due both to the convection and to the electrical contacts. As shown on Figure 8, the influence of electrical contacts is independent of temperature while the influence of convection increases with temperature. One can eventually limit these influences using long wires, however long wires require high volume samples which is not always possible. 


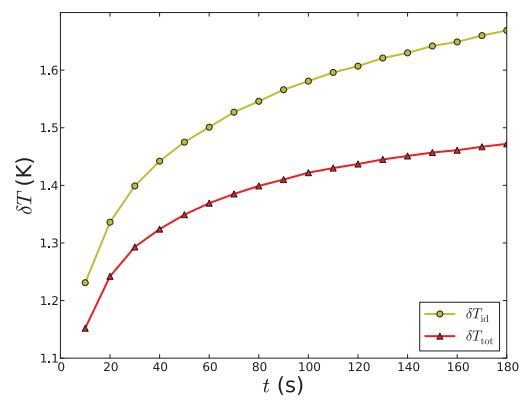

(a) Measurement temperature $T_{i}=293 \mathrm{~K}$. The Influence of convection is totally negligible, $\delta T_{\text {id }}>\delta T_{\text {tot }}=\delta T_{\text {ec }}$.

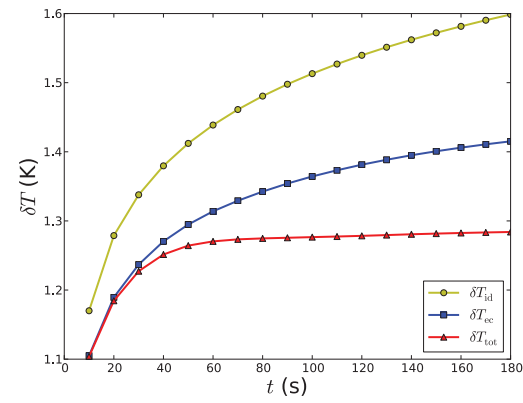

(b) Measurement temperature $T_{i}=373 \mathrm{~K}$. The Influence of convection is not negligible, $\delta T_{\text {id }}>$ $\delta T_{\text {tot }}$.

Fig. 8. Influence of the convection and electrical contacts on the temperature variation of a thin platinum wire immersed in glycerol within the framework of transient hot wire method.

\subsection{3 $3 \omega$ technique}

\subsubsection{Presentation}

The $3 \omega$ technique, introduced for the first time by Cahill, has been widely used for the characterization of dielectric thin films (Cahill, 1990; Franck et al., 1993; Moon et al., 1996). The adaptation of the method for liquids is relatively recent (Chen et al., 2004; Heyd et al., 2008; Oh et al., 2008; S. R. Choi \& Kim, 2008) but its use is increasingly common (Paul et al., 2010) in a variety of applications ranging from anemometry (Heyd et al., 2010) to thermal microscopy (Chirtoc \& Henry, 2008).

This method uses the same basic principle as the THW technique but replaces the constant current $i$ through the heater by a sinusoidally varying current $i(t)=\hat{I} \cos \omega t$ where $\omega=2 \pi v$. The heat rate per unit length dissipated by Joule effect in the line is written this time as $\dot{q}(t)=$ $\dot{q}_{\max }(1+\cos 2 \omega t) / 2$. This heat rate generates in the wire a temperature oscillation $\delta T(t)$ that contains a $2 \omega$ component $\delta T_{2 \omega}(t)=\delta \hat{T}_{2 \omega}^{0}(v) \cos 2 \omega t+\delta \hat{T}_{2 \omega}^{q}(v) \sin 2 \omega t$. Since the resistance of the wire is a known function of temperature, the voltage drop $u(t)=R(t) i(t)$ across the wire contains a $3 \omega$ component that can be written as $u_{3 \omega}(t)=\hat{U}_{3 \omega}^{0}(v) \cos 3 \omega t+\hat{U}_{3 \omega}^{q}(v) \sin 3 \omega t$. With the same experimental design as in the case of the transient method, one can use an appropriate synchronous detection to detect the quadrature components of $u_{3 \omega}(t)$ and derive then the thermal conductivity $k$ and specific heat $c_{m}$ of the fluid.

To go further into the analysis, we must express the temperature variation $\delta T(t)$ of the line heater by using the fundamental expression (16), but this time with $\dot{q}(t)=$ $\dot{q}_{\max }(1+\cos 2 \omega t) / 2$, where $\dot{q}_{\max }=R\left(T_{i}\right) \hat{I}^{2} / L$ :

$$
\begin{aligned}
\delta T(t) & =\frac{\dot{q}_{\max }}{8 \pi k} \int_{0}^{t}\left(1+\cos 2 \omega t^{\prime}\right) e^{-\frac{a^{2}}{4 \alpha\left(t-t^{\prime}\right)}} \frac{\mathrm{d} t^{\prime}}{t-t^{\prime}} \\
& =\delta T_{\mathrm{DC}}(t)+\delta T_{2 \omega}(t)
\end{aligned}
$$

The $\delta T_{\mathrm{DC}}(t)$ term corresponds to the transient method, (Par. 3.5.2.1) and will not be discussed here. Furthermore the synchronous detection allows to detect the electrical signals induced 


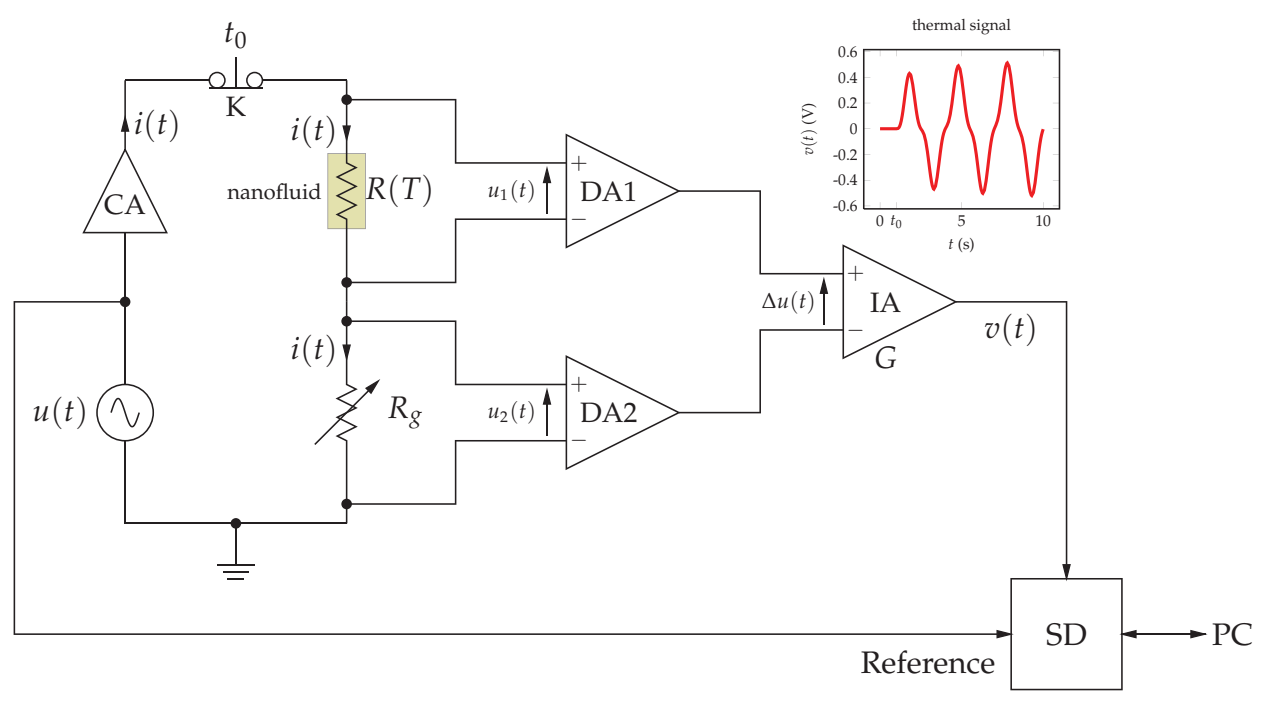

Fig. 9. $3 \omega$ circuit using a voltage divider and a dual phase DSP lock-in amplifier (SD) like model 7265 from Signal Recovery.

by $\delta T_{2 \omega}(t)$ insensitive to the influence of $\delta T_{\mathrm{DC}}(t)$. Using the $t \gg t_{\ell}$ usual approximation and introducing $\Lambda=\sqrt{\alpha / 2 \omega}$ the thermal length of the fluid, it can be shown (Hadaoui, 2010) that:

$$
\begin{aligned}
\delta \mathrm{T}_{2 \omega}(t) & =\frac{\dot{q}_{\max }}{4 \pi k} \Re\left[\mathrm{K}_{0}\left(\frac{a}{\Lambda} \mathrm{i}^{\frac{1}{2}}\right)\right] \cos 2 \omega t-\frac{\dot{q}_{\max }}{4 \pi k} \Im\left[\mathrm{K}_{0}\left(\frac{a}{\Lambda} \mathrm{i}^{\frac{1}{2}}\right)\right] \sin 2 \omega t \\
& =\delta \hat{T}_{2 \omega}^{0} \cos 2 \omega t+\delta \hat{T}_{2 \omega}^{\mathrm{q}} \sin 2 \omega t
\end{aligned}
$$

where $\Re$ and $\Im$ denote respectively the real and the imaginary part and $K_{0}$ is the modified Bessel function. The formatting (27) is not easy to use to analyze experimental datas. Using the following approximation:

$$
\lim _{x \rightarrow 0} \mathrm{~K}_{0}\left(x \mathrm{i}^{\frac{1}{2}}\right)=\ln 2-\gamma-\mathrm{i} \frac{\pi}{4}-\ln x
$$

it is possible to propose more suitable expressions of $\delta \hat{T}_{2 \omega}^{0}$ and $\delta \hat{T}_{2 \omega}^{\mathrm{q}}$ as long as $\Lambda \gg a$ :

$$
\begin{aligned}
& \delta \hat{T}_{2 \omega}^{0}=\frac{\dot{q}_{\max }}{4 \pi k}\left(\frac{1}{2} \ln \frac{2 \alpha}{a^{2}}-\gamma-\frac{1}{2} \ln \omega\right) \\
& \delta \hat{T}_{2 \omega}^{\mathrm{q}}=\frac{\dot{q}_{\max }}{16 k}
\end{aligned}
$$

To check the accuracy of the approximate expressions (29) and (30), we have gathered in Table 5 the values of $\Lambda$ at room temperature for some common materials and excitation frequencies. As we can see in this Table, in the case of most of the liquids (here water and glycerol) it is not possible to use (29) and (30) for excitation frequencies greater than $1 \mathrm{~Hz}$. This is the main limitation of this technique for the thermal characterization of nanofluids because on the one hand low excitation frequencies require very stable external conditions and on the other hand 
the measurements take a long time, allowing to the convection and to losses due to electrical contacts to occur.

\begin{tabular}{c||c|c|c|c|c}
\hline materials & water & air & glycerol & silicon & platinum \\
\hline$v_{0}=10^{-1} \mathrm{~Hz}, \Lambda=$ & 335 & 3981 & 271 & 8433 & 4469 \\
\hline$v_{0}=10^{0} \mathrm{~Hz}, \Lambda=$ & 106 & 1259 & 86 & 2667 & 1413 \\
\hline$v_{0}=10^{1} \mathrm{~Hz}, \Lambda=$ & 33 & 398 & 27 & 843 & 447 \\
\hline$v_{0}=10^{2} \mathrm{~Hz}, \Lambda=$ & 10,6 & 126 & 8,6 & 267 & 141 \\
\hline
\end{tabular}

Table 5. Values of the thermal length $\Lambda$ (in $\mu \mathrm{m}$ ) at RT for some common materials and usual excitation frequencies.

\subsubsection{Measurements}

As in the case of the Wheatstone bridge configuration, the voltage divider (Fig. 9) must be first balanced for each measurement temperature $T_{i}$ by ensuring that $R_{g}=R\left(T_{i}\right)$. The use of two differential amplifiers DA1 and DA2 (AMP03 like) allows to extract the informative signal. This signal $\Delta u(t)$ is a function of the temperature change $\delta T(t)$ of the line that is induced by Joule self-heating and heat exchanges with the fluid. As in the case of the transient technique, the amplitude of $\Delta u(t)$ is very small and needs to be amplified by a factor $G \approx 1000$ using an instrumentation amplifier (IA). The signal delivered by the amplifier includes a $3 \omega$ component that can be written as $v_{3 \omega}(t)=G u_{3 \omega}(t)$. Using relations (29) and (30), the amplitudes $X$ (in phase) and $Y$ (in quadrature) of the tension $v_{3 \omega}(t)$ can be written as:

$$
\begin{aligned}
& X(\omega)=\frac{\alpha_{w} R_{\mathrm{ref}} R\left(T_{i}\right) G \hat{I}^{3}}{8 \pi k L}\left(\frac{1}{2} \ln \frac{2 \alpha}{a^{2}}-\gamma-\frac{1}{2} \ln \omega\right) \\
& Y(\omega)=\frac{\alpha_{w} R_{\mathrm{ref}} R\left(T_{i}\right) G \hat{I}^{3}}{32 k L}=\mathrm{cst}
\end{aligned}
$$

As we can see from the relation (31), the amplitude $X(\omega)$ varies linearly with $\ln \omega$ and its theoretical graph is a straight line in a semi-log scale, with a slope $p_{X}=$ $-\alpha_{w} R_{\text {ref }} R\left(T_{i}\right) G \hat{I}^{3} / 16 \pi k L$. Once the physical properties of the experimental setup are precisely known, this expression allows for a very precise determination of the thermal conductivity $k$ by a frequency sweep of the exciting current $i(t)$ and measurements with a lock-in amplifier. As an example we have represented Fig. 10 the measurements obtained for pure glycerol at $T_{i}=298 \mathrm{~K}$. The value of the slope is $p_{X}=-0.0698$ which leads to a value of the thermal conductivity of the glycerol at RT: $k=0.289 \mathrm{~W} / \mathrm{mK}$.

\subsubsection{Comparison of the two techniques}

Both techniques are very similar because they are both derived from the hot wire method and have the same temporal and spatial limitations. The THW method has the advantage of being very fast but requires an important excitation $\delta T(t)$ which can cause significant errors primarily due to non-linearities and to influence of convection and electrical contacts.

In the case of liquids, the $3 \omega$ method requires measurement times significantly longer than those of the THW method. This can promote the influence of convection and electrical contacts. However the use of a very sensitive dual-phase synchronous detection allows for low-amplitude excitations within the $3 \omega$ framework, thus reducing the influence of non-linearities and spatial limitations. 


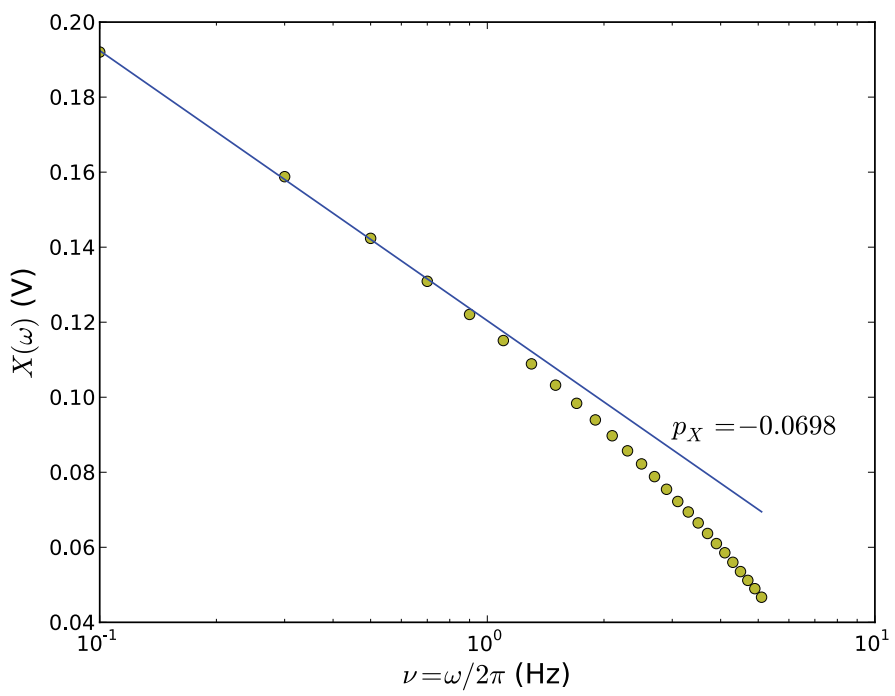

Fig. 10. Measurement of the thermal conductivity of pure glycerol at RT by the $3 \omega$ method. Experimental values: $R\left(T_{i}\right)=1.430 \Omega, R_{\text {ref }}=1.403 \Omega, G=993, L=2.5 \mathrm{~cm}$, $\alpha_{w}=3.92 \times 10^{-3} \mathrm{~K}^{-1}$ and $\hat{I}=148 \mathrm{~mA}$.

\section{Some basic rheological properties of nanofluids}

\subsection{Presentation}

The viscosity is probably as critical as thermal conductivity in engineering systems that use fluid flow (pumps, engines, turbines, etc.). A viscous flow dissipates mechanic power which volumic density is directly proportionnal to the dynamic viscosity $\eta$ of the fluid in the case of the laminar flow of a newtonian liquid.

As we have seen, the increase in thermal conductivity of nanofluids reaches values still incompletely explained, it is the same for the viscosity of these suspensions. The rheology of the nanofluids has given rise to much less research than the thermal behavior, and until now, the analysis of rheological properties of the nanofluids remains superficial. The predominance of the surface effects and the influence of aggregation are certainly the two major elements that distinguish a conventional suspension from a nanofluid, both from a thermal point of view than rheological.

\subsection{Experimental results}

Viscosity measurements concerning nanofluids generally do not obey directly to the classical models (Tab. 6) used to describe the behavior of the micro suspensions viscosity. Although the measures differ much from one study to another, as shwon on figure 11 common facts emerge and should guide future research:

- The size of NPs, that does not appear in the classical models, has an unpredicted influence on the viscosity of nanofluids. The shape of nanoparticles is another factor that may influence the rheology of the host liquid. Thus in most situations, spherical nanoparticles 
do not change the nature of a Newtonian fluid such as water or glycerol. In contrast the CNTs can dramatically change the nature of the liquid.

- The nature of the host liquid has a great influence on the law of variation of the relative viscosity $\eta_{r}=\eta / \eta_{0}$, where $\eta$ is the dynamic viscosity of the nanofluid and $\eta_{0}$ is the dynamic viscosity of the host fluid taken at the same temperature. For a given host liquide, the nature of nanoparticles with the same shape and with same size has very low influence on the dynamic viscosity of the suspension.

\begin{tabular}{|c|c|c|}
\hline Model & Expression & Comments \\
\hline Einstein & $\eta_{r}=1+[\eta] \phi+O\left[\phi^{2}\right]$ & $\begin{array}{l}\text { Effective medium theory for spherical particles and } \\
\text { dilute non-interacting suspensions }(\phi<10 \%) \text {. The } \\
\text { intrincic viscosity }[\eta] \text { has a typical value of } 2.5\end{array}$ \\
\hline Brinkman & $\eta_{r}=\frac{1}{\left(1-\phi^{2.5}\right)}$ & $\begin{array}{l}\text { Modified Einstein's equation to a more generalised } \\
\text { form. (Brinkman, 1952) }\end{array}$ \\
\hline Mooney & $\eta_{r}=\exp \left(\frac{\xi \phi}{1-k \phi}\right)$ & $\begin{array}{l}\text { Higher concentrations interacting spherical } \\
\text { suspensions. } k \text { is a constant called the } \\
\text { self-crowding factor (1.35<k<1.91), and } \\
\xi \text { is a fitting parameter chosen to agree with } \\
\text { Einstein's value of 2.5. (Mooney, 1951) }\end{array}$ \\
\hline Krieger-Dougherty & $\eta_{r}=\left(1-\frac{\phi}{\phi_{m}}\right)^{-[\eta] \phi_{m}}$ & $\begin{array}{l}\text { Interactions between neighboring spherical } \\
\text { particles are taken into account. } \phi_{m} \text { is the } \\
\text { maximum particle packing fraction and }[\eta]=2.5 \\
\text { for spherical particles. (Krieger \& Dougherty, 1959) }\end{array}$ \\
\hline Batchelor & $\eta_{r}=1+2.5 \phi+6.2 \phi^{2}$ & $\begin{array}{l}\text { Spherical particles and semi-dilute suspensions, } \\
\text { interaction of pair-particles are considered. } \\
\text { (Batchelor \& Green, 1972) }\end{array}$ \\
\hline
\end{tabular}

Table 6. Some classical models commonly used for viscosity of micro dispersions as a function of the volume fraction $\phi$ of solid particles. The relative viscosity is defined by $\eta_{r}=\eta / \eta_{0}$, where $\eta_{0}$ and $\eta$ are the dynamic viscosities respectively of the base liquid and of the suspension.

Dynamic Light Scattering (DLS) and cryo-TEM measurements in general show that nanoparticles agglomerate (He et al., 2007; Kwak \& Kim, 2005) in the liquid, forming micro-structures that can alter the effective volume fraction of the solid phase. This can be the main reason for the big difference between the viscosity behaviour of micro-suspensions and that of nano-suspensions. These observations suggest that, due to formation of micro-aggregates of nanoparticles, the effective volume fraction $\phi_{\text {eff }}$ of nanofluids can be much higher than the actual solid volume fraction $\phi$, which leads to a higher viscosity increase of nanofluids. Using an effective volume fraction that is higher than the initial solid fraction is a way to reconciliate observed results with those predicted by classical models. To justify that the aggregation of nanoparticles leads to an effective volume fraction higher than the initial fraction, some authors Chen et al. (2009) have introduced the fractal geometry to predict this increase in volume fraction. According to the fractal theory, the effective particle volume fraction is given by:

$$
\phi_{\text {eff }}=\phi\left(\frac{d_{\text {eff }}}{d}\right)^{3-D}
$$


$d$ and $d_{\text {eff }}$ are respectively the diameters of primary nanoparticles and aggregates, $D$ is the fractal index having typical values ranging from 1.6 to 2.5 for aggregates of spherical nanoparticles.

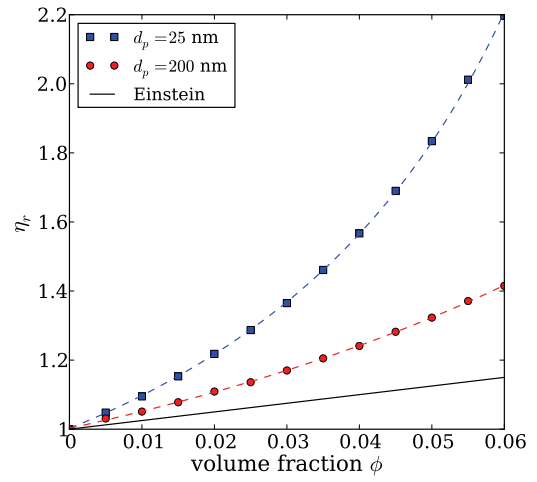

(a) Water-based nanofluids, adapted from (Corcione, 2011).

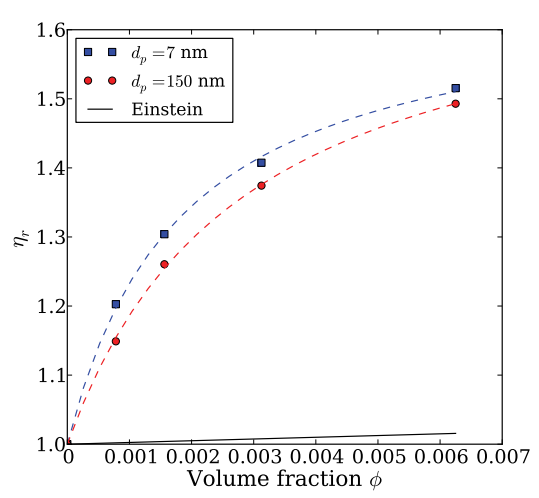

(b) Glycerol-based nanofluid, adapted from (Hadaoui, 2010).

Fig. 11. Evolution of viscosity as a function of $\phi$ and NPs diameter $d_{p}$.

Using a modified Krieger-Dougherty model where $\phi$ is replaced by $\phi_{\text {eff }}$ given by (33), it is possible to correctly describe measurements corresponding to a lot of different water-based nanofluids, as shown by dashed lines on Fig. 11(a). The same remark holds for glycerol-based nanofluids but using this time a modified Mooney model where $\phi$ has been replaced by $\phi_{\text {eff }}$ (dashed lines on Fig. 11(b)).

It is also very interesting to study the evolution of viscosity as a function of temperature. In the case of glycerol-based nanofluids containing spherical copper oxides NPs, we have found (Fig. 12) that the variation of the viscosity vs temperature always obeys a generalized Arrhenius law, regardless of the size and volume fraction of the NPs:

$$
\eta=A \exp \frac{B}{T-T_{0}}
$$

As shown on Fig. 12(a) and Tab. 12(b), the dependence of the viscosity with temperature is mainly due to the host fluid. This is reasonable because, as one might expect the loss of viscous fluid by friction on the NPs depends few on temperature, even if the fractal geometry of the micro-aggregates is certainly a function of temperature.

\subsection{Perspectives}

As we can see, the inclusion of nanoparticles in the host liquid can greatly increase the viscosity even at low volume fractions $(<1 \%)$. This increase may be a serious obstacle for many applications. In the field of lubrication, for example, an increased viscosity is an advantage for transmission of normal stresses but it is a disadvantage with regard to the friction forces that dissipate more energy within the liquid thereby increasing its temperature. 


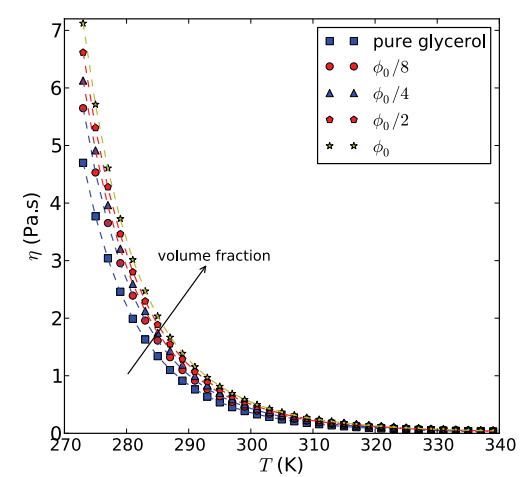

(a) Evolution of Glycerol-based nanofluids dynamic viscosity with temperature and volume fraction. $\phi_{0}=0.625 \%$.

\begin{tabular}{llll}
\hline$\phi$ & $A\left(10^{-7}\right.$ Pa.s $)$ & $B(\mathrm{~K})$ & $T_{0}(\mathrm{~K})$ \\
\hline 0 & 4.4 & 4.10 & 130 \\
$\phi_{0} / 8$ & 5.5 & 4.06 & 131 \\
$\phi_{0} / 4$ & 5.7 & 4.08 & 131 \\
$\phi_{0} / 2$ & 6.1 & 4.09 & 131 \\
$\phi_{0}$ & 6.5 & 4.10 & 130 \\
\hline
\end{tabular}

(b) Evolution of Arrhenius law coefficients as a function of NPs volume fraction.

Fig. 12. The variation ot the viscosity as a function of temperature $T$ follows an Arrhenius law regardless of the NPs volume fraction.

In the biomedical field, functionalized nanoparticles are used or intended for use as contrast agents for medical imaging or (and) grafted with therapeutic molecules used to kill cancer cells in a targeted manner (therapeutic nanocarriers). The hydrodynamic radius of these functionalized NP is often on average equal to almost $100 \mathrm{~nm}$, a value very close to those of aggregates encountered with nanofluids. It is therefore very probable that the results we have outlined above are relevant to predict the rheological behavior of blood products containing nanoparticles functionalized. To our knowledge there are no or very few studies that address the biophysical impact of functionalized NPs on the transport properties of human blood. This study is certainly critical to patient safety and should be considered both as a theoretical point of view using for example molecular dynamics simulations and as an experimental point of view through for example the use of the microfluidic devices available today.

\section{Conclusion}

The applications of nanofluids are numerous and very promising especially in the area of the transport of thermal energy. The wide dispersion of experimental results and numerical models available show that much remains to be done to identify clear trends and reliable models to describe the heat transfer at the scale of the nanoparticle. Even if the the role of the aggregation and secondarily the role of the ordered interfacial molecular layer seem preponderant in many situations, there is currently no comprehensive model allowing to predict the thermal behavior of all nanofluids. The lack of knowledge about the influence of surface treatment (the use of a polymer coating, the use of surfactants, the grafting of ions) on the heat transfer at the nanoparticle scale is certainly a major reason (by introducing unknown thermal interfacial resistances) of the dispersion of results and numerical models proposed until now. Systematic comparative studies and use of molecular dynamic simulations for transport coefficients modeling and Monte-Carlo simulations for aggregation modeling and control should allow progress on this subject. 
If the thermal conductivity is an important quantity for predicting heat transfer and as such has been widely studied, other physical properties and phenomena deserve further investigations like: the viscosity $\eta$, the heat transfer coefficient $h$, the specific heat $c_{m}$, nanocomposites changes of state, etc.

On the other hand the concerns of preservation of the nature should be considered more systematically, both at the level of green synthesis of nanoparticles than in terms of their composition. The multifunctional core-shell nanoparticles like $\mathrm{SiO}_{2} @ \mathrm{M}$ (where $\mathrm{M}$ is a metal) partially meet the previous requirements. They have already been studied for medical imaging and should also be considered for the transport of heat.

\section{References}

Abramowitz, M. \& Stegun, I. A. (eds) (1970). Handbook of Mathematical Functions, with Formulas, Graphs, And Mathematical Tables, ninth edn, Dover.

Batchelor, G. K. \& Green, J. T. (1972). The determination of the bulk stress in a suspension of spherical particles to order $c^{2}$, Journal Fluid Mechanics 56: 401-427.

Benoit, R., Warmont, F., Meynen, V., Witte, K. D., Cool, P., Delapierre, M. P. \& Saboungi, M.-L. (2009). Optimisation of the surface properties of sba-15 mesoporous silica for in-situ nanoparticle synthesis, Microporous and Mesoporous Materials 120: 2-6.

Brinkman, H. (1952). The viscosity of concentrated suspensions and solutions, Journal of Chemical Physics 20: 571-581.

Buongiorno, J., Venerus, D. C., Prabhat, N., McKrell, T. \& Townsend, J. (2009). A benchmark study on the thermal conductivity of nanofluids, Journal of Applied Physics 106: 14.

Cahill, D. (1990). Thermal conductivity measurement from 30 to $750 \mathrm{~K}$ : the $3 \omega$ method, Rev. Sci. Instrum. 61: 802.

Carslaw, H. \& Jaeger, J. (1959). Conduction of Heat in Solids, second edition.

Casquillas, G. V. (2008). Contrôle de température et étude des transferts thermiques dans des dispositifs microfluidiques, PhD thesis, Orsay Sciences Faculty, Paris.

Chen, F., Shulman, J., Xue, Y., Chu, C. W. \& Nolas, G. S. (2004). Thermal conductivity measurement under hydrostatic pressure using the $3 \omega$ method, Rev. Sci. Instrum. 75: 4578.

Chen, H., Witharana, S., Jin, Y., Kim, C. \& Ding, Y. (2009). Predicting thermal conductivity of liquid suspensions of nanoparticles (nanofluids) based on rheology, Particuology 7: 151-157.

Chen, Y., Peng, D., Lin, D. \& Luo, X. (2007). Preparation and magnetic properties of nickel nanoparticles via the thermal decomposition of nickel organometallic precursor in alkylamines, Nanotechnology 18.

Chirtoc, M. \& Henry, J. (2008). $3 \omega$ hot wire method for micro-heat transfer measurements: From anemometry to scanning thermal microscopy (sthm), Eur. Phys. J. Special Topics 153: 343.

Choi, S., Zhang, Z., Yu, W., Lockwood, F. \& Grulke, E. (2001). Anomalous thermal conductivity enhancement in nanotube suspensions, Applied Physics Letters 79: 2252-2254.

Choi, U. (1995). Enhancing thermal conductivity of fluids with nanoparticles, Technical Report FED, vol 231, ASME, New-York. 
Chon, C. H., Kihm, K. D., Lee, S. P. \& Choi, S. U. S. (2005). Empirical correlation finding the role of temperature and particle size for nanofluid $\left(\mathrm{Al}_{2} \mathrm{O}_{3}\right)$ thermal conductivity enhancement, Applied Physics Letters p. 153107.

Corcione, M. (2011). Empirical correlating equations for predicting the effective thermal conductivity and dynamic viscosity of nanofluids, Energy Conversion and Management 52: 789-793.

Darroudi, M., Ahmad, M., Abdullah, A., Ibrahim, N. \& Shameli, K. (2010). Effect of accelerator in green synthesis of silver nanoparticles, Int. J. Mol. Sci. 11: 3898-3905.

Daungthongsuk, W. \& Wongwises, S. (2007). A critical review of convective heat transfer of nanofluids, Renewable and Sustainable Energy Reviews pp. 797-817.

Eastman, J. A., Choi, S. U. S., Li, S., Yu, W. \& Thompson, L. J. (2001). Anomalously increased effective thermal conductivities of ethylene glycol-based nanofluids containing copper nanoparticles, Applied Physics Letters 78: 718-720.

Emami-Meibodi, M., Vafaie-Sefti, M., Rashidi, A. M., Amrollahi, A., Tabasi, M. \& Sid-Kalal, H. (2010). A model for thermal conductivity of nanofluids, Materials Chemistry and Physics 123: 639-643.

Evans, W., Prasher, R., Fish, J., Meakin, P., Phelan, P. \& Keblinski, P. (2008). Effect of aggregation and interfacial thermal resistance on thermal conductivity of nanocomposites and colloidal nanofluids, International Journal of Heat and Mass Transfer 51: 1431-1438.

Franck, R., Drach, D. \& Fricke, J. (1993). Determination of thermal conductivity and specific heat by a combined $3 \omega /$ decay technique, Rev. Sci. Instrum. 64: 760.

Govindaraj, A. \& Rao, C. N. R. (2002). Organometallic precursor route to carbon nanotubes, Pure Appl. Chem. 74: 1571-1580.

Hadaoui, A. (2010). Effects of size and concentration on the thermal and rheological properties of nanofluids, PhD thesis, Orléans University - France.

Hadaoui, A., Heyd, R., Saboungi, M., Meducin, F., Warmont, F., Aeziane, E., Flyiou, M. \& Koumina, A. (2009). Des polymères organiques pour la synthèse de nanoparticules métalliques, Les VI ièmes Journées sur les Polymères Organiques et leurs Applications (JPOA VI), Université Ibn Tofail.

He, Y. R., Jin, Y., Chen, H., Ding, Y., Cang, D. \& Lu, H. L. (2007). Heat rransfer and flow behaviour of aqueous suspensions of $\mathrm{TiO}_{2}$ nanoparticles (nanofluids) flowing upward through a vertical pipe, International Journal of Heat and Mass Transfer 50: 2272-2281.

Henderson, J. \& van Swol, F. (1984). On the interface between a fluid and a planar wall: theory and simulations of a hard sphere fluid at a hard wall, Mol. Phys. 51: 991-1010.

Heyd, R., Hadaoui, A., Ameziane, E., Saboungi, M., Guillot, S. \& Milosevic, I. (2008). Thermal properties of biocompatible ferrofluids by the $3 \omega$ method, MSNOW 2008 International Workshop, Nancy.

Heyd, R., Hadaoui, A., Fliyou, M., Koumina, A., Ameziane, L. E., Outzourhit, A. \& Saboungi, M. (2010). Development of absolute hot-wire anemometry by the $3 \omega$ method, Rev. Sci. Instrum. 81: 044901.

Iijima, S. (1991). Helical microtubules of graphitic carbon, Nature p. 354:56.

Keblinski, P., Phillpot, S., Choi, S. \& Eastman, J. (2002). Mechanisms of heat flow in suspensions of nano-sized particles(nanofluids), International Journal of Heat and Mass Transfer 45: 855-863. 
Knibbe, P. (1986). The end-effect error in the determination of thermal conductivity using a hot-wire apparatus, Int. J. Heat Mass Transfer 29: 463-473.

Krieger, I. M. \& Dougherty, T. J. (1959). A mechanism for non-newtonian flow in suspensions of rigid spheres, Transactions of The Society of Rheology 3: 137-152.

Kwak, K. \& Kim, C. (2005). Viscosity and thermal conductivity of copper oxide nanofluid dispersed in ethylene glycol, Korea-Australia Rheology Journal 17: 35-40.

Liu, X., Geng, B., Du, Q., Ma, J. \& Liu, X. (2007). Temperature-controlled self-assembled synthesis of $\mathrm{CuO}, \mathrm{Cu}_{2} \mathrm{O}$ and $\mathrm{Cu}$ nanoparticles through a single-precursor route, Materials Science and Engineering A 448: 7-14.

Masliyah, J. \& Bhattarjee, S. (2006). Electrokinetic and colloid transport phenomena, John Wiley and Sons.

Moon, I., Jeong, Y. \& Kwun, S. (1996). The $3 \omega$ technique for measuring dynamic specific heat and thermal conductivity of a liquid or solid, Rev. Sci. Instrum. 67: 29.

Mooney, M. (1951). The viscosity of a concentrated suspension of spherical particles, Journal of Colloid Science 6: 162-170.

Oh, D., Jain, A., Eaton, J. K., Goodson, K. E. \& Lee, J. S. (2008). Thermal conductivity measurement and sedimentation detection of aluminum oxide nanofluids by using the $3 \omega$ method, International Journal of Heat and Fluid Flow 29: 1456.

Paul, G., Chopkar, M., Manna, I. \& Das, P. (2010). Techniques for measuring the thermal conductivity of nanofluids: A review, Renewable and Sustainable Energy Reviews 14: 1913-1924.

Putnam, S. A., Cahill, D. G. \& Braun, P. V. (2006). Thermal conductivity of nanoparticle suspensions, Journal of Applied Physics 99: 084308-1-084308-6.

Ramnani, S., Biswal, J. \& Sabharwal, S. (2007). Synthesis of silver nanoparticles supported on silica aerogel using gamma radiolysis, Radiation Physics and Chemistry 76: 1290-1294.

Roca, A. G., Morales, M. P. \& Serna, C. J. (2006). Synthesis of monodispersed magnetite particles from different organometallic precursors, IEEE TRANSACTIONS ON MAGNETICS 42: 3025-3029.

Choi, S. R. \& Kim, D. (2008). Real-time thermal characterization of $12 \mathrm{nl}$ fluid samples in a microchannel, Rev. Sci. Instrum. 79: 064901.

Sakuma, H. \& Ishii, K. (2009). Gas flow sputtering: Versatile process for the growth of nanopillars, nanoparticles, and epitaxial thin films, Journal of Magnetism and Magnetic Materials 321: 872-875.

Singh, Y., Javier, J., Ehrman, S. H., Magnusson, M. H. \& Deppert, K. (2002). Approaches to increasing yield in evaporation/condensation nanoparticle generation, Journal of Aerosol Science 33: 1309-1325.

Sun, S., Zeng, H., Robinson, D. B., Raoux, S., Rice, M., Wang, S. X. \& Li, G. J. (2004). Monodisperse $\mathrm{MFe}_{2} \mathrm{O}_{4}(\mathrm{M}=\mathrm{Fe}, \mathrm{Co}, \mathrm{Mn})$ nanoparticles, J. Amer. Chem. Soc. 126: 273-279.

Temgire, M. K., Bellare, J. \& Joshi, S. S. (2011). Gamma radiolytic formation of alloyed ag-pt nanocolloids, Advances in Physical Chemistry 2011.

Wang, B., Li, H. \& Peng, X. (2002). Research on the heat-conduction enhancement for liquid with nano-particle suspensions, J. of Thermal Science 11: 214-219.

White, F. (1991). Viscous fluid flow, 2nd edn, McGraw-Hill. 
Yang, Y., Zhang, Z., Grulke, A., Anderson, W. \& G.Wu (2005). Heat transfer properties of nanoparticle-in-fluid dispersions (nanofluids) in laminar flow, International Journal of Heat and Mass Transfer 48: 1107-1116.

Yin, M., Willis, A., Redl, F., Turro, N. \& O'Brien, S. P. (2004). Influence of capping groups on the synthesis of $\gamma-\mathrm{Fe}_{2} \mathrm{O}_{3}$ nanocrystals, J. Mater. Res 19: 1208-1215.

Yu, C.-J., Richter, A., Datta, A., Durbin, M. \& Dutta, P. (2000). Molecular layering in a liquid on a solid substrate: an x-ray reflectivity study, Physica B 283: 27-31.

Zhang, X., Gu, H. \& Fujii, M. (2006). Experimental study on the effective thermal conductivity and thermal diffusivity of nanofluids, International Journal of Thermophysics 27: 569-580. 


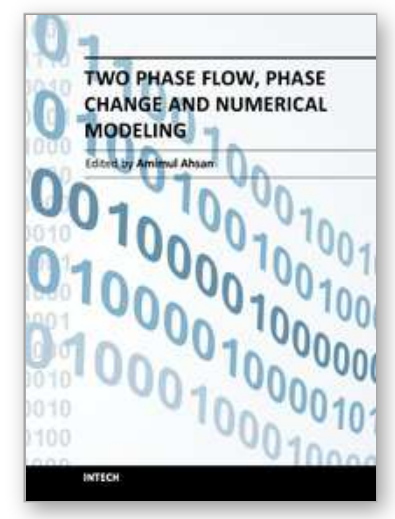

\author{
Two Phase Flow, Phase Change and Numerical Modeling \\ Edited by Dr. Amimul Ahsan
}

ISBN 978-953-307-584-6

Hard cover, 584 pages

Publisher InTech

Published online 26, September, 2011

Published in print edition September, 2011

The heat transfer and analysis on laser beam, evaporator coils, shell-and-tube condenser, two phase flow, nanofluids, complex fluids, and on phase change are significant issues in a design of wide range of industrial processes and devices. This book includes 25 advanced and revised contributions, and it covers mainly (1) numerical modeling of heat transfer, (2) two phase flow, (3) nanofluids, and (4) phase change. The first section introduces numerical modeling of heat transfer on particles in binary gas-solid fluidization bed, solidification phenomena, thermal approaches to laser damage, and temperature and velocity distribution. The second section covers density wave instability phenomena, gas and spray-water quenching, spray cooling, wettability effect, liquid film thickness, and thermosyphon loop. The third section includes nanofluids for heat transfer, nanofluids in minichannels, potential and engineering strategies on nanofluids, and heat transfer at nanoscale. The forth section presents time-dependent melting and deformation processes of phase change material (PCM), thermal energy storage tanks using PCM, phase change in deep $\mathrm{CO} 2$ injector, and thermal storage device of solar hot water system. The advanced idea and information described here will be fruitful for the readers to find a sustainable solution in an industrialized society.

\title{
How to reference
}

In order to correctly reference this scholarly work, feel free to copy and paste the following:

Rodolphe Heyd (2011). Nanofluids for Heat Transfer, Two Phase Flow, Phase Change and Numerical Modeling, Dr. Amimul Ahsan (Ed.), ISBN: 978-953-307-584-6, InTech, Available from:

http://www.intechopen.com/books/two-phase-flow-phase-change-and-numerical-modeling/nanofluids-for-heattransfer

\section{INTECH}

open science | open minds

\section{InTech Europe}

University Campus STeP Ri

Slavka Krautzeka 83/A

51000 Rijeka, Croatia

Phone: +385 (51) 770447

Fax: +385 (51) 686166

www.intechopen.com

\section{InTech China}

Unit 405, Office Block, Hotel Equatorial Shanghai

No.65, Yan An Road (West), Shanghai, 200040, China 中国上海市延安西路65号上海国际贵都大饭店办公楼 405 单元

Phone: +86-21-62489820

Fax: +86-21-62489821 
(C) 2011 The Author(s). Licensee IntechOpen. This chapter is distributed under the terms of the Creative Commons Attribution-NonCommercialShareAlike-3.0 License, which permits use, distribution and reproduction for non-commercial purposes, provided the original is properly cited and derivative works building on this content are distributed under the same license. 